BABELAO 4 (2015), p. 25-59

(C) ABELAO (Belgium)

\title{
Le thème de la révolte de l'objet contre son créateur dans la Bible et ses précurseurs dans les fables de la tablette KBo 32.14 écrite en langue hourrite
}

Par

\author{
Arnaud Fournet \\ La Garenne Colombes, France
}

\footnotetext{
T a tablette KBo 32.14 fait partie d'un lot de documents mis au jour pendant les campagnes de fouilles 1983 et 1985 à Boğazköy. Les tablettes sont bilingues et écrites à la fois en hourrite et en hittite, ce qui leur donne une très grande valeur pour la connaissance et la compréhension du hourrite, souvent handicapées par des textes monolingues difficiles à élucider. Leur étude montre qu'il s'agit d'originaux hourrites traduits en hittite. Le texte original et sa traduction se font face, respectivement sur les colonnes de gauche et de droite. Parfois le texte hittite suit le texte hourrite. Cette disposition se rencontre sur la face et le revers de toutes les tablettes, sur deux et parfois quatre colonnes, et elle est en soi une indication implicite que le texte hourrite est premier. Des lignes vides sont également ajoutées dans le texte hourrite pour éviter que les paragraphes ou les phrases ne soient décalés entre les deux langues. Il apparait ainsi que le texte hittite s'efforce de suivre le plus fidèlement possible l'original en hourrite
} 
tant dans le contenu que la mise en forme. Il faut néanmoins noter qu'à la fin de la tablette KBo 32.14, la traduction hittite suit l'original hourrite.

La copie autographe des tablettes a été publiée en $1990^{1}$ et une étude détaillée a été réalisée par $\mathrm{E}$. $\mathrm{Neu}^{2}$. Une traduction existe en langue anglaise, mais écrite par des hittitologues, de sorte qu'elle reflète plutôt le texte hittite. Par ailleurs une présentation ainsi qu'une étude partielle a été écrite en italien par S. de Martino ${ }^{4}$. J'ai aussi publié une étude des colonnes hourrites ${ }^{5}$. De façon plus distante, on peut également citer des études sur la transmission de la tradition épique du Proche-Orient ancien vers l'Anatolie et la Grèce. $^{6}$

L'article qui suit est une première en langue française. ${ }^{7}$ Outre l'étude proprement philologique des Fables, nous voulons mettre en perspective plus spécialement certains passages de la Bible, qui font référence à des thèmes littéraires et à des oeuvres populaires, que l'auditoire du proche-orient ancien ne pouvait manquer de connaître, à n'en pas douter.

Tout d'abord, il convient de séparer les différentes oeuvres regroupées dans le fascicule KBo 32, qui n'ont en commun que la date de leur découverte et le fait d'être bilingue.

\section{Oeuvre unique ou lots de tablettes?}

L'ensemble des tablettes, et des fragments, est généralement appelé chant de la libération, ou Song of Release, canto delle liberazione ou en allemand Epos der Freilassung, qui est le titre retenu par Neu. Plusieurs tablettes mettent en effet en scène la « libération » de la ville d'Ebla par le dieu Teššub. Il s'agit en fait d'une destruction

${ }^{1}$ H. OTTEN et Chr. RÜSTER, Keilschrifttexte aus Boghąköi (KBo), XXXII, 1990, Berlin.

${ }^{2}$ E. NeU, Das hurritische Epos der Freilassung (StBo 32), 1996, Wiesbaden.

${ }^{3}$ H. A. HoffNer Jr, Hittite Myths, 19982, Atlanta, pp. 65-80. La tablette KBo 32.14 occupe les pp. 68-73.

${ }^{4}$ S. de MARTINO, " Il canto delle liberazione », dans La civiltà dei Hurriti. La Parola del Passato, Vol. 55. Napoli, 2000, pp. 298-320.

5 A. Fournet, The Aesopic Fables in Hurrian, 2013, Lille.

${ }^{6}$ Cf. les travaux de M. R. Bachvarova, par exemple: «The Eastern Mediterranean Epic Tradition from Bilgames and Akeka to the Song of Release to Homer's Iliad», dans Greek, Roman, and Byzantine Studies 45, 2005, pp. 131-153.

${ }^{7}$ Comme son titre l'indique, J. CATSANiCOS, «L'apport de la bilingue Hattuša à la lexicologie hourrite ", dans J-M. DuRAND (ed.), Amurru 1, 1996, Paris, pp. 197-296, n'est pas philologique à proprement parler. Hoffner (op. cit. n. 3) ne le liste d'ailleurs pas dans sa bibliographie. 
plutôt qu'une libération. Le texte hittite porte l'idéogramme SìR 'chant' en sumérien, que Neu a préféré traduire Epos 'épopée', et le verbe hittite parà tarnumar 'libérer, émanciper', ce qui justifie le titre retenu. Ce sont les habitants (esclaves ou captifs) d'Ebla qui sont libérés plutôt que la ville, le refus de les émanciper étant la cause ultime de la destruction de la ville.

Le texte a sans doute comme origine la disparition historique d'Ebla, que l'on s'accorde à situer au $17^{\mathrm{e}}$ siècle av. J.C. ${ }^{8}$ Ce cadre est intéressant car il donne une borne temporelle pour la datation linguistique des tablettes qui prennent cet évènement comme thème littéraire. Incidemment, on peut soutenir que cette Epopée de la libération contient des éléments de propagande politique, puisque la destruction d'Ebla était le résultat de l'expansion hittite vers le sud à l'époque de Hattušili I et Muršili I. Au lieu d'être une "cause », la libération des habitants peut être considérée comme un prétexte rétroactif justifiant les projets d'expansion en Syrie. ${ }^{9}$

Le cunéiforme a été paléographiquement daté de la période médio-hittite, vers $-1400^{10}$. Naturellement cela ne prouve pas, bien au contraire, que le contenu des tablettes retrouvées partage cette datation. Car il s'agit de copies postérieures à la création, dans des proportions qui peuvent se chiffrer en siècles.

Certaines tablettes sont numérotées : $32.11\left(1^{\text {ère }}\right), 32.12\left(2^{\text {ème }}\right)$ et $32.15\left(5^{\text {ème }}\right)$. Cette dernière indique en outre : ul qati 'non terminé', de sorte que la série devait comporter au moins 6 tablettes. La tablette 32.16 s'avère être un duplicata de la 32.15, les deux tablettes cassées complétant une partie de leurs lacunes. Il circulait plusieurs exemplaires de la série entière. Un point dont on ne peut sous-estimer l'importance est que les tablettes 32.12 et 32.14 n'ont aucun lien thématique avec les autres, qui chantent la libération d'Ebla. De fait Neu aboutit à la conclusion que la 32.12 $\left(2^{\text {ème }}\right)$ n'appartient pas à la même série que la 32.11 (1 $\left.1^{\text {ère }}\right)$ et qu'elle fait suite à une autre tablette, tête de série, qui pour l'heure est inconnue. Il y a donc deux lots de tablettes : une série relative au Chant de la libération et un lot de Paraboles. Ainsi :

"Immerhin muß man aber damit rechnen, daß Tafel II [32.12] einer anderen Tafelserie angehörte und eine andere VorgängerTafel hatte."11

${ }^{8}$ Cf. E. NEU, op. cit. (n. 2), p. 2.

${ }^{9}$ Cf. S. de MArTino, op. cit (n. 4), p. 298. "poteva [...] essere anche utilizzato a livello propagandistisco da tale sovrano, sostenitore di una politica di expansione verso l'Anatolia sud-orientale e la Siria ».

${ }^{10}$ IDEM, p. 3.

${ }^{11}$ IDEM, p. 17. "Après tout, cependant, il faut aussi compter sur le fait que la tablette II [la 32.12] appartenait à une autre série et avait une autre tablette [que la 32.11] comme tablette précédente." 
Ce point de vue est également mentionné par de Martino ${ }^{12}$. Il mérite d'être développé. Il ne s'agit pas d'une simple hypothèse de travail mais d'une réalité qu'il convient de décrire et d'apprécier.

Les tablettes 32.12 et 32.14 doivent être étudiées à part. Par ailleurs il faut noter que si la série de (6?) tablettes du Chant de la libération forme une oeuvre cohérente, rien ne prouve que le lot de Paraboles 32.12 et 32.14 forme une série continue. On ne peut pas écarter l'hypothèse que ces deux tablettes soient indépendantes, même si elles appartiennent au même genre littéraire.

\section{Le cas de la tablette 32.14}

La suite s'intéresse plus particulièrement aux colonnes de la tablette 32.14 écrites en langue hourrite. Il existe plusieurs raisons de penser que ce texte n'a pas de lien avec le Chant de la libération et que sa langue et donc aussi sa composition sont nettement plus anciennes :

1. En premier lieu la thématique de la 32.14 n'a aucun rapport avec la ville d'Ebla. Il s'agit de paraboles ou de fables, plus ou moins morales, qui n'ont pas d'ancrage géographique et ne font pas - en tout cas très peu - intervenir de divinités.

Du point de vue littéraire, ces Fables sont des précurseurs des Fables d'Esope et, plus tard, des Fables de la Fontaine.

2. La partie hourrite ne contient quasiment aucun idéogramme suméro-akkadien et l'écriture est purement phonétique.

3. Les emprunts akkadiens sont assez rares. Le vocabulaire est très majoritairement hourrite, contrairement à d'autres tablettes où l'on rencontre en moyenne presque un mot akkadien par ligne, et parfois même plus. Il a été proposé que le Chant de la libération aurait un prototype syrien. Il faut néanmoins noter que certains passages de la tablette 32.14 contiennent une densité d'emprunts akkadiens significativement plus forte que les autres, ce qui est certainement une information sur leur origine.

4. Enfin, la morphologie verbale dans la tablette 32.14 est très singulière, en décalage par rapport à celle du hourrite classique, tel qu'il transparait dans la Lettre du Mittanni ou les tablettes du Chant de la libération. Ce point a déjà fait l'objet de différentes discussions et il est nécessaire d'y revenir dans la suite de l'article. Il n'existe qu'un seul exemple du suffixe de présent transitif -iya dans tout le texte (ligne 23 revers), dans un passage qui ressemble à une greffe plus tardive que le reste du texte.

\footnotetext{
12 de MARTINO, op. cit (n. 4), p. 301. “[...] appartenessero ad una serie
} differente da quella di [...]." 
5. La morphologie nominale semble également archaïque : ABL -dani, voire -ni au lieu de -dan, GEN -bi au lieu de -wi. Il est fait un usage parsimonieux de l'article défini -ni.

6. Hoffner remarque que les autres Chants hourrites, tels que le cycle de Kumarbi, ne contiennent pas de paraboles. ${ }^{13}$ Pourtant il maintient qu'il s'agit d'une oeuvre unifiée. ${ }^{14}$

7. La tablette 32.14 ne recourt pas au verbe sirad- 'chanter', visiblement adapté du sumérien SÌR 'chant', mais à kad- 'parler'. ${ }^{15}$

Pour toutes ces raisons, thématiques, graphiques, lexicales et morphologiques, il nous semble que la tablette 32.14 doit être étudiée indépendamment et ne fait pas partie de l'épopée en vers relative à Ebla. Ces paraboles sont narrées, racontées (kad-) et non pas chantées (sirad-).

\section{Structure générale de la tablette 32.14}

Maintenant que l'autonomie des fables est établie, il convient d'en décrire la structure littéraire et thématique, ainsi que leur origine, proprement hourrite ou akkadienne.

En tout, la tablette $\mathrm{KBo} 32.14$ comprend les parties suivantes :

- fable 1 du chevreuil et de la montagne, (1-16)

- fable 2 de l'homme et de la ville, (17-22)

- paragraphe intercalaire avant la fable suivante (23-25)

- fable 3 du chevreuil et de l'autre pâture, (26-30)

- fable 4 de l'homme et de l'autre forteresse, (31-38)

- paragraphe intercalaire avant la fable suivante (39-41)

- fable 5 de la coupe et du fondeur, (42-60)

- fable 6 du fils et du père, (1-5)

- paragraphe intercalaire avant la fable suivante (6-8)

${ }^{13}$ H. A. HoffNer Jr, op. cit. (n. 3), p. 66. «Unlike this composition they [the Kumarbi myths] contained no parables. »

${ }^{14}$ IDEM. « It may be justly asked why the entire text is presented in an anthology of [Hurro-]Hittite myths. The answer is that, although we see diverse genres, the Hurrian and Hittite scribes saw a unified work. » Il y a quelque chose de tout à fait fallacieux à attribuer ce point de vue aux scribes hourrites et hittites. La vérité est qu'ils n'ont rien dit de tel. Incidemment on peut noter que Hoffner ne dit pas un mot des problèmes de numérotation des tablettes, notés par Neu et qui vont à l'encontre de son point de vue.

${ }^{15}$ H. A. HOFFnER Jr (op. cit. n. 3, p. 67) considère à tort que siradsignifie 'to tell' [dire] : «I will tell (siratili)». 
- fable 7 du chien voleur, (9-12)

- fable 8 de l'administrateur voleur, (13-19)

- paragraphe intercalaire avant la fable suivante (20-22)

- fable 9 du kami (loir?) voleur, (23-25)

- fable 10 du maire voleur, (26-27)

- paragraphe intercalaire avant la fable suivante (33)

- fable 11 de la tour et du bâtisseur, (35-40)

- fable 12 du fils et du père, (48-49) [=fable 6]

- paragraphe intercalaire avant la fable suivante (53)

- fable 13 du bois et de l'empileur, (55-61)

- fable 14 de l'apprenti et du maittre, (63-65)

D'après leur concentration en emprunts akkadiens, qui est soit quasi-nulle soit importante, on peut assigner l'origine suivante à chacune des fables :

- fable 1 : hourrite,

- fable 2 : hourrite,

- fable 3 : hourrite,

- fable 4 : fort substrat akkadien,

- fable 5 : essentiellement hourrite avec un mot akkadien,

- fable 6 : hourrite,

- fable 7 : fort substrat akkadien, avec connotations obscènes,

- fable 8 : hourrite, mais avec un sens politique,

- fable 9 : fort substrat akkadien, avec connotations obscènes,

- fable 10 : fort substrat akkadien,

- fable 11 : fort substrat akkadien, avec morphologie hybride,

- fable 12 : hourrite,

- fable 13 : fort substrat akkadien dans la première phrase,

- fable 14 : hourrite,

Ces fables sont pour la moitié des traductions en hourrite, plus ou moins saturées en mots akkadiens. Hormis les fables 7 et 9, qui ressemblent à des chansons à boire, les fables à fort substrat akkadien sont plus politiques et plus techniques que celles en hourrite. A l'inverse, hormis la fable 5 à contenu métallurgique, une spécialité anatolienne, les fables hourrites se réfèrent à la nature : chevreuil, montagne, chasseur, bois ou à des liens sociaux de base : fils-père, homme-ville, apprenti-maitre.

On peut également noter que les passages les plus répétitifs, qui rythment la rhétorique et qui raccordent les fables entre elles, sont clairement en hourrite, et généralement en hourrite (très) archaïque.

Cet ensemble de fables est une composition qui intègre et recycle des éléments préexistants: fables hourrites, fables akkadiennes et chansons à boire traduites. Il semble que la date de l'assemblage ne soit pas ancienne, même si les matériaux élémentaires qui ont nourri cet ensemble sont certainement anciens. Un indice clair qui trahit une action peu ancienne sur le 
texte se trouve dans la ligne 23 revers : kamenes kakkari taliya ephenidan 'Le kami prend un gateau kakkari dans le four'. C'est le seul exemple de présent -iya dans toutes les formes verbales du texte, pourtant nombreuses et variées. A juger d'après le témoignage morphologique des autres lignes, si la ligne 23 était ancienne, elle ressemblerait à kameneš kakkari tala ephenidani ou peut-être même kameneš kakkeari tala epheni.

Il faut donc sans doute conclure que la composition et l'assemblage final de la tablette KBo 32.14 sont contemporains du hourrite classique du Mittanni à partir d'items préexistants dont la langue n'a pas été modernisée.

\section{La révolte de l'objet contre son créateur}

Les fables d'Esope, que recellent les tablettes KBo 32.12 et 32.14 découvertes en 1983-85, ont une valeur inestimable, car il s'agit d'un genre littéraire, qui était très populaire dans l'antiquité mésopotamienne, mais qui fut complètement ignoré par les écoles scribales. Nous savons que ce genre de fables, mettant en scène des animaux ou des choses qui portraiturent et caricaturent des personnes et des traits de caractère humain, existait mais il n'en a pas été retrouvé d'exemplaires en akkadien.

Le lot de fables KBo 32 aborde différents défauts : le manque de gratitude envers ceux qui nourrissent et élèvent, la révolte contre son créateur ou son géniteur, la cupidité et la trahison, etc. Il ne fait pas de doute que les peuples du proche-orient ancien connaissaient ce type de fables. Une partie des fables en hourrite sont d'ailleurs des traductions quasi certaines. C'est dans ce cadre qu'il est intéressant de mettre en parallèle certaines d'entre elles avec des passages de la Bible.

Concernant plus précisément le thème de la révolte contre son créateur, Hoffner ${ }^{16}$ fait remarquer en passant que des paraboles similaires figurent dans la Bible ${ }^{17}$ avec des poteries et des potiers, mais il ne cite pas les sources et ne développe pas la question du parallélisme :

Jérémie 18:1-6: La parole qui s'adressa à Jérémie de la part du SEIGNEUR : «Descends tout de suite chez le potier; c'est là que je te ferai entendre mes paroles. » Je descendis chez le potier ; il était en train de travailler au tour. Quand, par un geste malheureux, le potier ratait l'objet qu'il confectionnait avec de l'argile, il en refaisait un autre selon la technique d'un bon potier. Alors, la parole du SEIGNEUR s'adressa à moi : Ne puis-je pas agir avec vous, gens d'Israël, à la manière de ce potier ? - oracle du SEIGNEUR. Vous

\footnotetext{
${ }^{16}$ Cf. H. A. Hoffner Jr (op. cit. n.3, p.70).

${ }^{17}$ Nous citons la traduction dite oecuménique.
} 
êtes dans ma main, gens d'Israël, comme l'argile dans la main du potier.

Lettre aux Romains 9:19-21: Qui es-tu donc, homme, pour entrer en contestation avec Dieu? L'ouvrage va-t-il dire à l'ouvrier: Pourquoi m'as-tu fait ainsi ? Le potier n'est-il pas maitre de son argile pour faire, de la même pâte, tel vase d'usage noble, tel autre d'usage vulgaire?

Esaïe 29:16 : «Quel renversement des rôles! Prendra-t-on le potier pour l'argile? L'oeuvre dira-t-elle de l'ouvrier: «Il ne m'a pas faite »? Le vase dira-t-il du potier : «Il n'y entend rien »?

Esaïe 49:9 : "Malheur à qui, cruchon parmi les cruchons de glaise, chicanerait celui qui l'a formé ! L'argile dira-t-elle à celui qui lui donne forme : «Que fais-tu ?», et l'oeuvre réalisée par toi dirat-elle : «Il n'a pas de main»?

La morale implicite est évidemment que la chose créée n'est pas censée se rebeller contre son créateur, renier le lien de filiation ou se croire plus intelligente que lui. Cette morale transparait dans plusieurs fables de la tablette 32.14, que nous allons examiner dans la suite de l'article.

\section{Les lignes 1-16 de la tablette 32.14 col. I}

La première fable illustre le thème de l'ingratitude, plutôt que celui de la révolte, mais sa structure est très proche des autres.

La tablette d'origine, publiée en copie autographe dans (KBo 32.14 p.7), présente sensiblement cette apparence :

[haut de tablette]

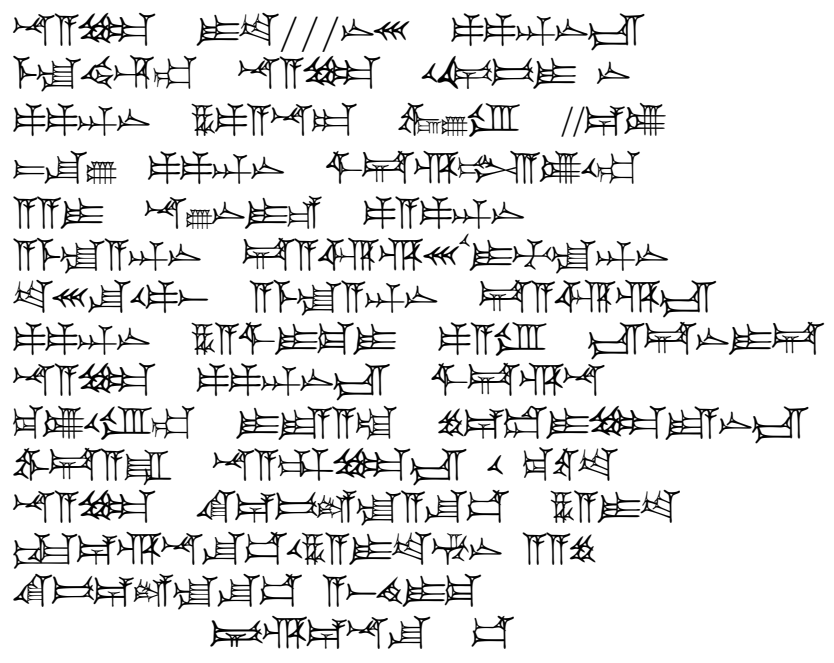

Après numérotation des lignes, translitération (TC) et essai de restitution en hourrite classique normalisé $(\mathrm{HCN})$ on aboutit à la situation suivante : 


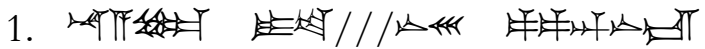
TC na-a-la ${ }^{18}$ i-te-////-no-es pa-pa-an-no-iš

$\mathrm{HCN}$ nali edi[ye]nišspabanniš

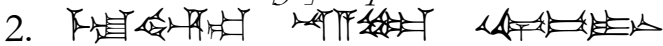

TC me-la-ab-bu ${ }^{19}-u m^{20}$ na-a-la u-ul-bi-i-na

HCN melabbum nali ulßini

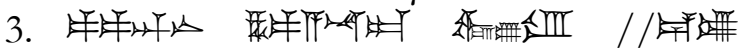

TC pa-pa-an-nə ba-pa-a-na-ab wo wi- $^{-}-r u / /{ }^{21}-e-l u$

HCN pabanni habanab ßuru(b) [t]elu

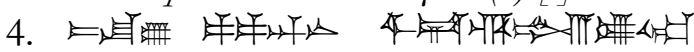

TC tab-šs-ú pa-pa-an-na ši-ta-ro-il-lu-u-um

HCN tapšu pabanni šidarillum

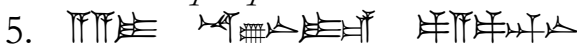

TC a-a-i na-ú-nə-i-e pa-a-pa-an-no

$\mathrm{HCN}$ ai nauniye pabanni

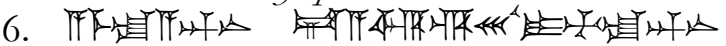

TC a-me-la-a ${ }^{22}$-an-na ta-a-ar-ra-es $u^{23}$ i-ti-la-an-na

$\mathrm{HCN}$ amelanni tarriš u idelanni

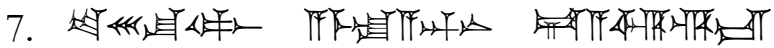

TC te-eš-su-u-ba-aš a-me-la-a-an-no ta-a-ar-ro-iš

$\mathrm{HCN}$ Tešsubaš amelanni tarriš

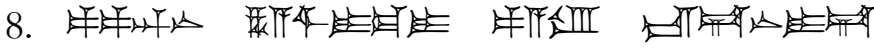

TC pa-pa-an-nə ha-a-sici-i-ma-i pa-a-ru iš-ta-nə-i-ta

HCN pabanni hašmae paru [b] ištani(yi)da

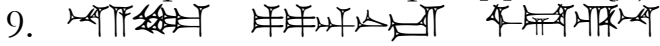

TC na-a-la pa-pa-an-no-iš ši-ta-ar-na

HCN nali pabannis šidarn [iy] a

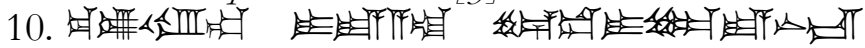

TC ku-lu-u-ru-um i-ia-a-at še-e-du-i-la-ia-no-iš

HCN kulurum iyat(ta) seduiliyanis

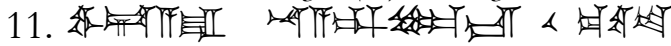

TC si-ta-a-ra na-a-al-b-is u $u^{24}$ ku-ut-te

HCN sidara [b] nalliš u kutte

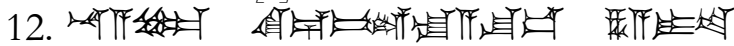

TC na-a-la ke ${ }^{25}-e-b i-i l-l a-a-s ̌ u-u s^{2}$ ba-a-i-te

HCN nali kebillašuš baide

${ }^{18} \mathrm{Il}$ n'y a pas de raison de cacher le fait que certaines signes ont des valeurs multiples et qu'on ne sait pas exactement s'il faut lire $l i$ ou $l e$.

${ }^{19}$ Le signe Tah est tronqué et ligaturé avec le suivant, ce qui donne l'impression que hu est manquant:

${ }^{20} \mathrm{Neu}(\mathrm{StBo} T 35$, p.74) note ici $<:>$ qui n'existe pas.

${ }^{21}$ Le signe est presque entièrement effacé mais compatible avec te.

22 Partiellement effacé.

${ }^{23} \mathrm{Neu}($ StBoT35, p.74) remplace le signe $<u$ par $<:>$.

${ }^{24} \mathrm{Neu}(\mathrm{StBoT} 35, \mathrm{p} .74)$ remplace le signe $<u$ par $<:>$.

25 Signe bivalent à lecture ke et ki. 


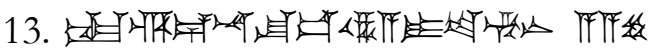

TC ka-re-e-na-šsu-uš $u^{26}$ ba-a-i-te-en-no ${ }^{27} a-a-\check{s}^{2} e$

$\mathrm{HCN}$ karen (n)ašus haidenni aře

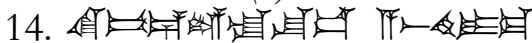

TC ka-bi-e-il-la-šu-us a-aś-bi-i-ma

HCN kebillašuš ašhima(e)

15.

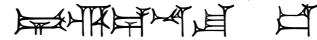

TC ga-re-e-na-šs- us

$\mathrm{HCN}$ karen $(n) a \check{s}^{2} \dot{s}^{28}$

Vocabulaire :

$a i$ 'si'

am- 'brûler, incendier', existe aussi en ourartéen amašt-,

aše 'chair, viande'

ašh- 'dépiauter, faire la peau'

edi 'corps'

ha- 'saisir, capturer'

baban- 'se rendre, se déplacer'

haš- 'entendre',

id- 'casser en morceaux',

iśtani 'intérieur, milieu'

iya 'pourquoi'

kareni 'oiseleur'

kebili 'chasseur'

kud- 'tuer, abattre'

kul- 'dire', kulur- 'dire maintes fois'

melabh- 'chasser, éloigner'

nali 'chevreuil, cerf, existe aussi en akkadien,

nauni 'pâturage'

pabani 'montagne', existe aussi en ourartéen baban,

par- 'se mettre en colère'

šedu- 'engraisser',

šid- 'maudire', šidar- 'maudire à maintes reprises'

tapšu' 'en bas'

tari 'feu'

telu 'en haut',

$u$ 'et, et puis, alors', sans doute un emprunt akkadien,

ulßi 'autre',

Bur- 'voir'

La tablette sépare les mots avec des blancs mais on a affaire à un paragraphe complet sans ponctuation interne. Pour segmenter en propositions et phrases il faut s'appuyer sur différents indices, tels que les conjonctions, $u$ et $i y a$, les formes verbales, à finale $-m$ et $-b$, les marques de cas, en particulier l'ERG. On aboutit à la structure et ponctuation suivantes :

\footnotetext{
${ }^{26} \mathrm{Neu}($ StBoT35, p.74) remplace le signe $\propto u$ par $<:>$.

${ }^{27} \mathrm{Neu}(\mathrm{StBoT} 35, \mathrm{p} .74)$ remplace en-nə par $e n_{6}$.

${ }^{28}$ La ligne 16 est vide du côté hourrite.
} 
Nali edi[ye]niš pabanniš melahbum, nali ulßini pabanni habanab.

Buru[b] [t]elu tapšsu pabanni sidarillum:

«Ai nauniye pabanni amelanni tarriš

u idelanni Tešsubaš, amelanni tarriš !»

Pabanni hasimae paru [b] istani(yi)da,

nali pabannišs sidarn [iy]a kulurum:

«Iyat(ta) šeduiliyaniš šidara[b] nalliš ?

u kudde nali kebillašus !

baide karen [n]ašušs!

baidenne aše kebillašsuš !

ašbima[e] karen [n]ašuš !»

Analyse $^{29}$ : Nali edi[ye] niš pabanniš melabhum,

Nali 'chevreuil' à l'ABS., objet du verbe melabh-, edi[ye]nišs 'son corps' à l'ERG. DEF, épithète de pabannis, pabannis 'la montagne' à l'ERG. DEF, sujet du verbe melahb-, melabhum 'chasser, expulser', au GER,

Un chevreuil d'elle-même la montagne ayant chassé, ${ }^{30}$

Analyse : nali ulßini pabanni habanab.

Nali 'chevreuil' à l'ABS., sujet du verbe de mouvement haban-, ulßini 'l'autre' à l'ABS. DEF, épithète de pabanni, pabanni 'la montagne' à l'ABS. DEF, en fonction de LOC., habanab 'se rendre, se déplacer', au PRET archaïque, [le dit] chevreuil en l'autre montagne se rendit.

Analyse : Buru[b] [t]elu tap̌ru pabanni šidarillum:

Buru [b] 'voir, regarder', sans finale $-b$ ou $-m$. Ce verbe est logiquement intransitif (finale $u$ ) mais pourrait représenter un présent. Après un début au passé, il semble que le texte se poursuive au présent, ce qui expliquerait l'absence de $-b$,

telu 'en haut', nom positionnel à finale $-u$,

tapš́ 'en bas', nom positionnel à finale $-u$,

pabanni 'la montagne' à l'ABS. DEF, objet du verbe šidar-, šidarillum 'se mettre à maudire à maintes reprises', au GER,

Il regarda de haut en bas, se mettant à maudire la montagne à maintes reprises:

Dans la traduction en hittite, le passage $\beta u r u[b]$ [t]elu tapš́ est rendu par 'il chercha querelle', mais ce n'est pas le sens littéral du texte hourrite : 'regarder de haut en bas'. Cette expression semble similaire à 'prendre de haut, chercher noise'. Plusieurs passages du texte montre que les verbes hourrites amul- et $\beta u r$ ' 'regarder' ne sont pas seulement factuels mais impliquent aussi un jugement de valeur: 'bien ou mal considérer'.

29 Je suis les principes précédemment présentés dans A. FOURNET, «Eléments de morphologie et syntaxe de la langue hourrite », dans Babelao, 2013, vol. 2 pp. 3-52.

${ }^{30}$ H. A. HOFFNER Jr (op. cit. n.3, p.78) prétend que le texte hourrite est : «A roe-deer — its favorite [sic] mountain chased it away. » 
Si on interprète $\beta u r u[m]$ comme un GER, alors il faut rattacher cette phrase à la précédente et traduire regardant de haut en bas, commençant à la maudire à maintes reprises. En l'absence de marque formelle, les deux analyses sont possibles.

Par ailleurs Neu considère que le chevreuil maudit la montagne qu'il vient de quitter [den ehemaligen Berg] mais rien ne l'indique en vérité. Cela peut tout à fait être la montagne sur laquelle il s'est rendu. ${ }^{31}$

Analyse : "Ai nauniye pabanni amelanni tarris

$A i$ 'si', conjonction,

nauniye 'son pâturage', à l'ABS. avec Poss P3Sg, sujet, ${ }^{32}$

pabanni 'la montagne' à l'ABS. DEF, en fonction de LOC., amelanni 'incendié', PART au DESI, prédicat adjectival, tarris ‘par le feu', à l'ERG. DEF, exprimant le moyen, " S'il advient que $[\mathrm{m}]$ on pâturage en la montagne soit incendié par le feu, Le texte hourrite est en fait au style indirect.

Analyse : u idelanni Teřšrubaš, amelanni tarriš !»

u'alors', conjonction,

idelanni 'détruit, cassé', PART au DESI, prédicat adjectival,

Těšsubaš 'par Teššub, à l'ERG. DEF, exprimant le moyen, amelanni 'incendié', PART au DESI, prédicat adjectival, tarriš 'par le feu', à l'ERG. DEF, exprimant le moyen, alors, je veux qu'elle soit cassée par Tě̌šub [et] incendiée par le feu!»

Analyse : Pabanni hasimae paru[b] istani(yi)da,

pabanni 'la montagne' à l'ABS. DEF, sujet du verbe par-, hašimae 'en entendant [cela]', PART à l'INST., paru $[b]$ 'être en colère', PRET sans finale $-b$ ou $-m$, iśtani(yi)da 'en son [for] intérieur', à l'ALL. L'ALL. exprime peutêtre l'acquisition par opposition au LOC. qui exprimerait l'état. La montagne, en entendant [cela], se mit en colère en son [for] intérieur,

Analyse : nali pabanniš sidarna kulurum:

Nali 'chevreuil' à l'ABS., objet du verbe sidarn-, pabannis 'la montagne' à l'ERG. DEF, sujet du verbe šidarn-, sidarna 'maudire',

kulurum 'dire maintes fois', au GER,

Et la montagne de maudire maintes fois le chevreuil, en répétant:

L'interprétation de sidarna comme un nom au pluriel laisse nali sans fonction syntaxique, c'est pourquoi je préfère y voir un verbe, mais la finale $-a$ est étrange, on attendrait plutôt un PRET

31 H. A. HoffNer Jr (op. cit. n.3, p.78) penche pour la nouvelle et indique avoir discuté cette question par lettre avec Neu, qui a maintenu son point de vue initial. Hoffner (p.69) cite plusieurs autres auteurs qui considèrent tous que « the deer cursed the new mountain. »

${ }^{32}$ H. A. HOFFnER Jr (op. cit. n.3, p.69) interprète nauniye comme un participe du type śeduiliya mais sa traduction : «the mountain on which I am grazing » est fautive, puisque dans ce cas on devrait avoir naun-iya-ni. 
transitif du type -ib. Le même problème se pose dans la phrase suivante. Dans son interprétation principale Neu tient sidarna pour un nom au pluriel: " $\mathrm{Zu}$ dem Rehbock sprach der Berg Flüche» [la montagne dit des malédictions au chevreuil] et nali pour un datif ${ }^{33}$, mais cette approche est à mon avis difficile voire impossible. L'un des mots doit être un verbe transitif et ce ne peut être que šidarna. Cf. ligne 46.

Analyse : «Iyat [ta] šeduiliyaniš šidara nalliš ?

Iya 'pourquoi', conjonction, -t, PEM P1Sg-(a)tta, objet du verbe TRA šidar-, šeduiliyaniš 'engraissé', PART à l'ERG. DEF, épithète de nali, sidara 'maudire',

nallis' 'chevreuil' à l'ERG. DEF, sujet du verbe šidar-,

Pourquoi le cheurenil, engraissé [par moi], me maudit-il ?

La forme sidara, avec une finale - $a$ dans une forme conjuguée, semble exclure la diathèse transitive, mais la phrase contient un objet -atta et un sujet nallis. Une variante (archaïque ?) en - $a$ du PRES sidariya est une hypothèse possible. Neu traduit sidara par le présent verbal : verflucht [maudit]. ${ }^{34}$

Analyse : u kudde nali kebillašus !

u'alors', conjonction,

kudde 'abattre, tuer', verbe à l'OPTA $(<\mathrm{HCN} *$ kud-eden $))^{35}$

nali 'chevreuil' à l'ABS., objet du verbe TRA kut-,

kebillašnš 'les chasseurs', à l'ERG. DEF, sujet du verbe kut-, ${ }^{36}$

Alors, qu'ils abattent le chevremil, les chasseurs!

Analyse : haide karen [n]ašuš !

baide 'saisir, capturer', verbe à l'OPTA ( $<\mathrm{HCN} * b a-i-e d e n)$, karen [n] ašuš 'les oiseleurs', à l'ERG. DEF, sujet du verbe ba-, qu'ils le capturent, les oiseleurs!

Analyse : haidenne ăse kebillašuš !

baidenne 'saisir, capturer', verbe à l'OPTA (< $\mathrm{HCN} *$ ba-i-eden $)$, àse 'viande, chair' à l'ABS., objet du verbe $h a-$,

33 Sans exclure que "nali [bildete] dann dazu das Objekt » dans une autre hypothèse. (p.110)

${ }^{34}$ Cf. la suggestion de Neu dans ce sens (p.112) : «Zum Ergativ nälli-š „der Rehbock” gehört die transitive Verbalform ši-ta-a-ra $(\stackrel{s i d}{=}=a r=a)$, deren auslautendes $-a$ als Endung (3. Pers. Sing.) anzusprechen ist [...] fragt man

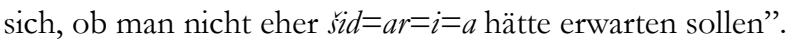

${ }^{35}$ La phonétique de la base kud- (ou kut- ?) n'est pas claire. Ailleurs on trouve la forme kudu avec une sonore mais dans $<$ ku-ut-te $>$ on devrait avoir une sourde $[t]$. Cette forme repose sur le contact de deux occlusives sonores: $* k u d+(e) d e(n)$. Il semble improbable que le résultat soit une sourde, il faut donc conclure qu'il s'agit alors d'une vraie géminée *[kudde], à la jonction de deux morphèmes kud-de(n). Cf. ligne 57 kudu.

36 Les pluriels définis kebillašuš et karenašuš reposent sur une forme courte de l'article -nna avec un seul $n:{ }^{*} n a-$. 
kebillašsuš 'les chasseurs', à l'ERG. DEF, sujet du verbe kut-, qu'ils saississent sa chair, les chasseurs!

Analyse : ašbima[e] karen [n]ašuš !»

ašbima [e] 'dépiauter', PART à l'INST.,

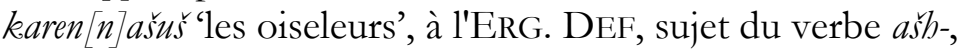
en le dépiautant, les oiseleurs!»

Traduction continue :

Nali edi[ye]niš pabanniš melabum,

Un cheurenil d'elle-même la montagne ayant chassé,

nali ulßini pabanni habanab.

[le dit] chevreuil en l'autre montagne se rendit.

Buru[b] [t]elu tapšsu pabanni sidarillum:

Il regarda de haut en bas [chercha noise], se mettant à mandire la montagne à maintes reprises:

«Ai nauniye pabanni amelanni tarriš

« S'il advient que $[\mathrm{m}]$ on pâturage en la montagne soit incendié par le feu,

u idelanni Těšsubaš, amelanni tarriš !»

alors, je veux qu'elle soit cassée par Těšsub [et] incendiée par le feu!»

Pabanni hašimae paru [b] istani(yi)da,

La montagne, en entendant [cela], se mit en colère en son [for] intérieur,

nali pabannišs sidarn [iy] a kulurum:

Et la montagne de maudire le chevreuil, en répétant:

«Iyat [ta] šeduiliyanišs sidar [iy] a nalliš?

Pourquoi le chevrenil, engraissé [par moi], me maudit-il ?

u kutte nali kebillašus! !

Alors, qu'ils abattent le chevreuil, les chasseurs!

baide karen [n]ašuš !

qu'ils le capturent, les oiseleurs!

haidenni aše kebillašuš!

qu'ils saississent sa chair, les chasseurs!

ašbima [e] karen [n]ašuš! »

en le dépiautant, les oiseleurs!»

Il s'agit donc d'une sorte de conte ou de fable, qui narre un chevreuil s'attirant des ennuis en proférant des malédictions irresponsables contre la montagne qui le nourrit. Le sens général est une critique de l'ingratitude ou de l'arrogance. Les lignes suivantes (17-22) offrent un parallèle humain. Cela explique aussi sans doute qu'un objet en théorie inanimé comme une montagne puisse être au cas ergatif dans les lignes (1-16). 


\section{Les lignes $42-51$ de la tablette 32.14 col. I}

Ces lignes contiennent la cinquième fable, qui illustre à la perfection le thème du potier et de l'argile, du fondeur et de la coupe. Et on peut utilement comparer avec les citations d'Esaïe vues ci-dessus au $₫ 4$.

Ce paragraphe (KBo 32.14 p.8) présente sensiblement cette apparence :

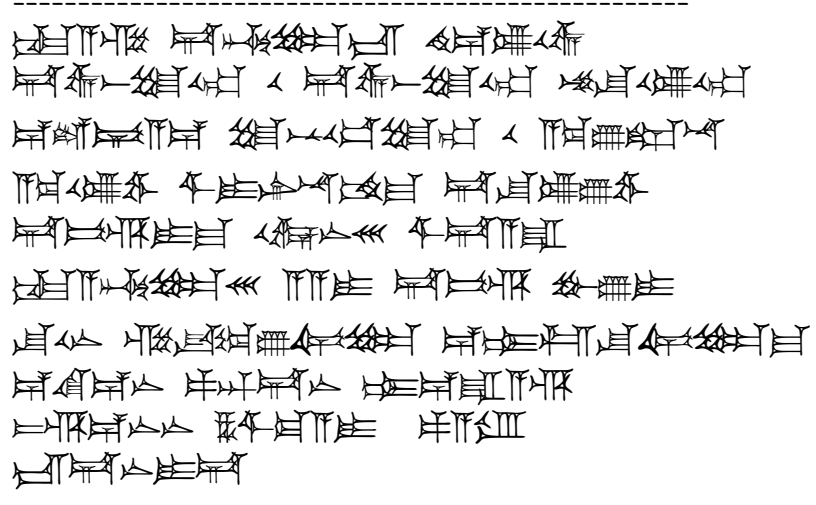

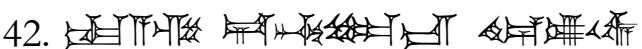

TC ka-a-zo ta-bal-ba-is bo-e-lu-u-wo a

HCN kazi taballis heluwa

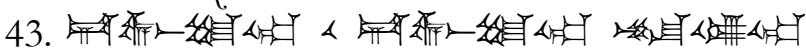

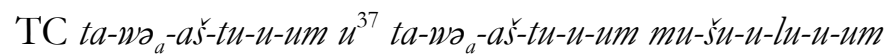

HCN tawastum $u$ tawastum musulum

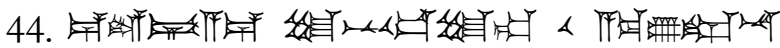

TC e-i $]^{8}-g a-a-e$ tu-nu-u-uś-tu-um $u^{39}$ a-ku-ú-úr-na

$\mathrm{HCN}$ elgae tunustum u agurna

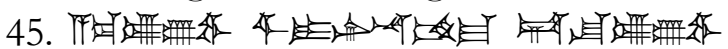

TC $a-k u-l u-u ́-w \partial^{40} \quad \grave{s i}-i-\partial r-n a-a m-m a$ ta-šu-lu-ú-wa ${ }^{41}$

HCN agulub(i) ${ }^{42}$ sirnamma tašulub(i)

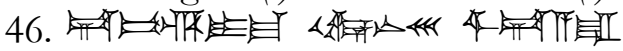

TC ta-bi-ro-i-ma u-wo-no-es si-ta-a-ra

HCN tabirima ußenes sidara

${ }^{37}$ Neu remplace ce signe par $<:>$.

${ }^{38}$ De façon étonnante, le signe $e$, différent de $i$, est suivi du signe il, pourtant différent de $\langle$ sill el. Cf. ligne 57 e-el-ka et 58 e-il-ka.

${ }^{39} \mathrm{Neu}$ remplace ce signe par $<:>$.

${ }^{40} \mathrm{Neu}$ lit wa mais le signe n'est pas désambiguïsé quant à la voyelle.

${ }^{41} \mathrm{Neu}$ lit wa mais le signe n'est pas désambiguïsé quant à la voyelle.

${ }^{42}$ Cf. lignes 28, 35 pour la même question de lecture de ce signe. 


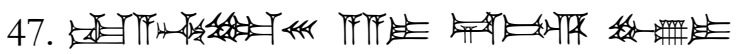

TC ka-a-bal-la-eśs a-a-i ta-bi-ro pu-ú-i

HCN kaballeš ai tabiri Buwi

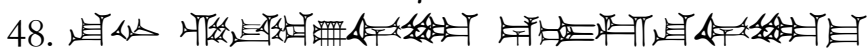

TC šu-u-na za-ak--ku-úul-la e-hé-ab-šu-ul-la-ma

HCN šni agkeulli ${ }^{43}$ ehepsullima

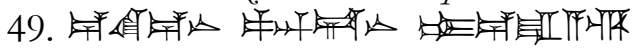

TC e-ka-e-na pa-an-ta-na bé-e-ra-a-ra

$\mathrm{HCN}$ egen(n)i $\beta$ andani herari

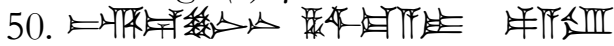

TC tab-ra-e-in ${ }^{44}$-na ba-ši-ma-a-i pa-a-ru

HCN tabrenni hašimae paru

51.

TC iś-ta-no-i-ta

$\mathrm{HCN}$ istani(yi)da

Vocabulaire nouveau :

agul- 'ciseler, sculpter'

aguri 'ciselure, sculpture'

elgi 'placage brillant'

ege(n)ni 'intérieur, interne' < egi 'intérieur',

ehepšsul- 'se coincer, se pincer'

hel- 'parler'

belu 'gloire, réputation'

herari 'tendon'

kabali 'cuivre',

ka(z) zi 'coupe, tasse', < akkadien kasu, cf. arabe ka'su, mušul- 'bien former, donner la bonne forme'

$\beta u(w i)$ 'de toi (GEN. arch. au lieu de $\beta$ ewi $)^{45}$

širnami 'lumière, éclat'

suni 'main'46

tabali, tabiri 'fondeur, métallurgiste'

taß-, taßašt- 'fondre, couler (un métal)'

tašul- 'donner'

tunust- 'plaquer, enduire'

uße 'insensé, idiot'

Banda(n)ni [Adjectif] 'droit(e)' < $\beta$ andi [Nom] 'côté droit'

zokk- 'casser', z̧k.kul- 'se casser'

La segmentation en phrases est la suivante :

Kaz̨i taballiš heluwa tawaštum,

u taßastum mušulum elgae tunuštum,

u agurna agulub(i) šrnamma tašulub(i).

${ }^{43} \mathrm{Neu}$ considère que ce verbe doit se lire qikkkul-.

${ }^{44} \mathrm{Neu}$ lit $e n_{6}$. A noter que $\mathrm{e}$ est suivi par in alors que le signe en existe.

${ }^{45}$ Cf. la finale de prétérit archaïque - $b$ et $a b i$ 'qui ?'.

${ }^{46}$ Ressemble au sumérien $\check{s} \mathfrak{u}$ 'main' avec un suffixe individualisant - $n i$. 
Tabirima ußeneš sidar(iy) a kaballeš :

" ai tabiri, Buwi suni zokekulli,

ehepšullima egen(n)i ßandan(n)i herari!»

Tabrenni hašimae paru ištani(yi)da.

Analyse : Kazi taballiš heluwa taßaštum,

kaqi 'coupe', à l'ABS., COD de taß(aśt)-,

taballis' 'fondeur', à l'ERG. DEF., sujet de ta $\beta($ aśt)-,

heluwa 'gloire', au DAT.,

taßaštum 'fondre', au GER,

Une coupe le fondeur ayant coulée pour (sa) réputation,

Analyse : u taßaštum mušulum elgae tunuštum,
$u$ 'et (puis)'
taßastum 'fondre', au GER,
mušulum 'bien former', au GER,
elgae 'placage brillant', à l'INST., s
tunustum 'appliquer', au GER,
Analyse: $u$ agurna agulub(i) sirnamma tašulub(i).
$u$ 'et (puis)'
agurna 'ciselure', à l'ABS. DEF. PL, COD de agul-, agulub(i) 'ciseler', au PRET archaïque,
sirnam- 'éclat', à l'ABS. DEF. SG, COD de tašul-,
- ma 'et', conjonction ENC,
tašulub(i) 'donner', au PRET archaïque,
et puis il cisela des ciselures et [lui] donna de l'éclat.

et la coulant en la formant bien [et lui] appliquant une couche brillante,

Analyse : Tabirima ußeneš šidar(iy)a kaballě̌ :

tabiri- 'fondeur', à l'ABS. sans article, COD de sidar-, - ma 'et', conjonction ENC,

ußeneš 'insensé', à l'ERG. DEF., déterminant de kabali,

sidar(iy)a 'maudire',

kaballeš 'cuivre', à l'ERG. DEF., sujet de sidar-,

Et le cuivre insensé de mandire le fondeur:

Analyse : «ai tabiri ßuwi suni zəkekulli,

ai 'si', conjonction,

tabiri 'fondeur', à l'ABS. sans article,

Buwi 'de toi', GEN. arch. au lieu de $\beta e w i,{ }^{48}$

šnni 'main', à l'ABS., sujet de zakkul-,

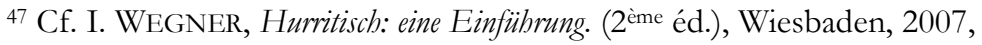
pp. 216-220, qui propose une analyse alternative de segmentation helub-a, avec un suffixe -ub-attesté dans turubi 'étranger, ennemi'. A noter que Wegner laisse les incohérences vocaliques entre $i$ et $e$ dans sa transcription (p. 216).

48 Wegner (p. 219) traite $\beta$ uwi comme une variante formelle du pronom possessif P1SG -iwnu-wi, ce qui est contredit par le blanc entre tabiri et $\beta$ swi. L'idée est néanmoins intéressante sémantiquement : si la main de mon fondeur. 
z̧okeulli 'casser', au passif et à l'OPTA : -ulli $<*$-ul-eni,

"[Et,] fondeur, si ta main se brise,

Analyse : ehepšullima egeni ßandani herari!»

ehepšsulli- 'se coincer', au passif et à l'OPTA : -ulli $<*$-ul-eni, - ma 'et', conjonction ENC,

egen(n)i 'interne', épithète de herari, Bandan $(n) i$ 'droit(e)', épithète de herari, herari 'tendon', sujet de ehepšnl-,

[alors] que [ton] tendon interne droit se coince!»

Analyse : Tabrenni hašimae paru ištani(yi)da. (cf. ligne 8)

tabrenni 'fondeur', à l'ABS. DEF.,

Le fondeur, en entendant [cela], se mit en colère en son [for] intérieur.

Traduction continue :

Kazi taballiš heluwa taßaštum,

Une coupe le fondeur ayant coulée pour (sa) réputation,

u taßaštum mušulum elgae tunuštum,

et la coulant en la formant bien [et lui] appliquant une couche brillante,

u agurna agulub(i) sirnamma tašulub(i).

et puis il cisela des ciselures et [lui] donna de l'éclat.

Tabirima ußeneš sidar(iy)a kaballeš :

Et le cuivre insensé de maudire le fondeur:

"ai tabiri ßuwi šni zokkulli,

" [Et,] fondeur, si ta main se brise,

ehepšullima egeni ßandani herari!»

[alors] que [ton] tendon interne droit se coince!»

Tabrenni hašimae paru ištani(yi) da.

Le fondeur, en entendant [cela], se mit en colère en son [for] intérieur.

\section{Les lignes $52-60$ de la tablette 32.14 col. I}

Le paragraphe suivant de la tablette (KBo 32.14 p.8) présente sensiblement cette apparence :

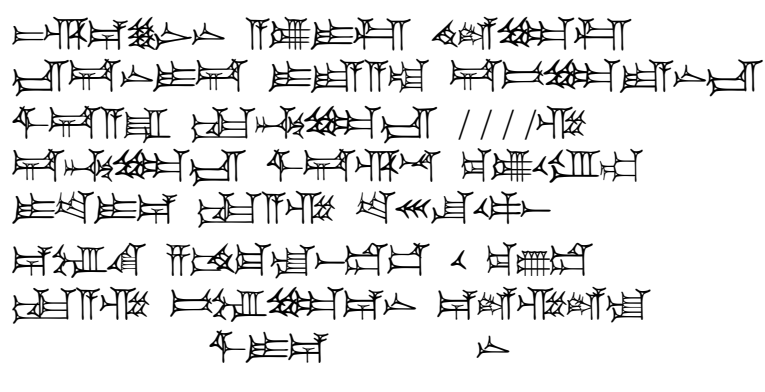




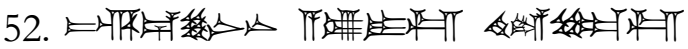

TC tab-ro-e-in ${ }^{49}$-na a-lu-i-ab bo-il-la-ab

HCN tabrenni aluib billi ${ }^{50}$

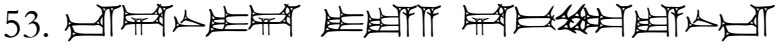

TC is-ta-na-i-da i-ia-a(t) ta-bi-lo-ia-nə-iš

$\mathrm{HCN}$ istani(yi)da iya(t) tabaliyanis

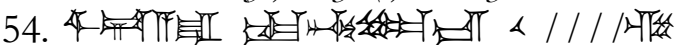

TC si-ta-a-ra ka-bal-la-iš $u^{51} \quad[k a-a]^{52}-z a$

HCN sidar(iy)a kaballis u kazi

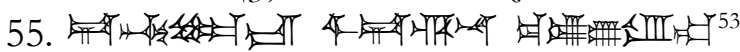

TC ta-bal-bo-iš ši-ta-ar-na ku-lu-ú-ru-um

$\mathrm{HCN}$ taballis sidarna kulurum

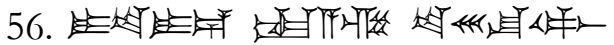

TC i-te $e^{54}-i-e$ kea-a-zo te-ers-šsu-u-pa-as

$\mathrm{HCN}$ idiye kazi Teřšrubas

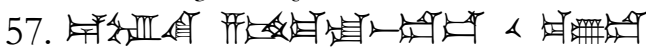

TC e-el-ka za-am-ma-la-aš-du-uš $u^{55} \quad k u-u-d u$

HCN elgi zammalaštuš u kudu

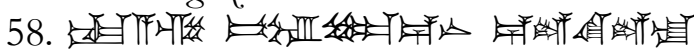

TC ka-a-za bi-el-la-e-na e-il-ka-il-la ${ }^{56}$

$\mathrm{HCN}$ kari pilleni elgilla

59.

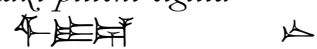

TC $\stackrel{s i-i-e}{ }$

$-n>$

$\mathrm{HCN}$ sizeni

Ce paragraphe est le dernier de la colonne I de KBo 32.14 mais il se poursuit sur le revers de la tablette en colonne IV.

Vocabulaire nouveau :

alu- 'parler'

belli 'parole'

pilli 'canal'

zammal-(ašt-) 'arracher',

La segmentation en phrases est la suivante :

Tabrenni aluib hilli istani(yi)da:

"Iya(t) tabaliyanis sidar(iy)a kaballis? ?"

U kari taballis sidarna kulurum:

${ }^{49}$ A noter l'incohérence -e-in-. Neu transcrit -e-en $\sigma_{-}$mais Wegner-e-in-

${ }^{50}$ Le signe -ab est apparemment une erreur. Cf. la ligne 38 du revers.

${ }^{51}$ Neu transcrit $u$ par $<:>$.

${ }^{52}$ Ces deux signes sont partiellement effacés mais reconnaissables.

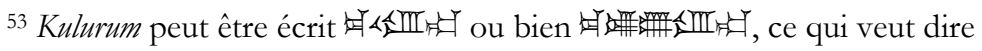
que $\propto$ et notent la même voyelle.

${ }^{54} \mathrm{Neu}$ transcrit te par $t i_{7}$, essayant de gommer les incohérences entre les voyelles.

${ }^{55}$ Neu transcrit $u$ par $<:>$.

${ }^{56}$ Cette ligne contient deux incohérences : bi-el- et $e-i l$. 
"Idiye kazi Teššbaš, elgi zammalaštuš !

u kudu kazi pilleni elgilla siyeni! »

Analyse : Tabrenni aluib billi ištani(yi)da:

tabrenni 'fondeur', à l'ABS. DEF., sujet de alu-, aluib 'parler', au PRET archaïque, ${ }^{57}$

helli 'parole', à l'ABS. DEF., COD de alu-, ištani(yi)da 'en son [for] intérieur', à l'ALL.,

Le fondeur se dit cette parole en son for intérieur:

Analyse : «Iya(t) tabaliyaniš šidar(iy)a kaballiš ? 》

Iya 'pourquoi', conjonction,

-(a)t, PEM P1Sg-(a)tta, objet du verbe TRA sidar- ${ }^{58}$

tabaliyanis 'fondu', PART à l'ERG. DEF, épithète de kaballi,

sidar(iy)a 'maudire', 59

kaballiš'cuivre' à l'ERG. DEF, sujet du verbe šidar-, "Pourquoi le cuivre que j' ai fondu me mandit-il ? "

Analyse : U kazi taballiš šidarna kulurum : ${ }^{60}$

$U$ 'et puis', conjonction,

kazi 'coupe', à l'ABS., COD de šidarn-,

taballiš 'la montagne’ à l'ERG. DEF, sujet du verbe šidarn-,

sidarna 'maudire',

kulurum 'dire maintes fois', au GER,

Et puis le fondeur de maudire la coupe en répétant:

Analyse : "Idiye kazi Teššbaš, elgi zammalaštuš

Idiye 'briser', à l'OPTA,

kazi i'coupe', à l'ABS., COD de $i d$-,

Teššbas 'Teššub' à l'ERG. DEF, sujet du verbe $i d$-, elgi 'applique brillante', à l'ABS., sujet de zammalašt-, zammalaštus' 'arracher complètement', au GER passé,

${ }^{57} \mathrm{La}$ forme aluib ressemble à un prétérit archaïque. Si on ne supprime pas le $b$ final de billi $b]$ on a alors une sorte de pléonasme : aluib hillib 'il parla et dit'. La ligne 38 du revers incite à le supprimer. Par ailleurs la présence d'un $u$ dans aluib est un peu étrange et s'explique mal.

${ }^{58} \mathrm{Ce}$ pronom n'est pas explicite, sans doute à cause de l'identité avec l'initiale du mot suivant. Il est présent dans la ligne 38 du revers.

${ }^{59}$ Wegner (p. 231) considère que - $a$ est un « Personenanzeiger 3. Pers. Sg. trans. ».

${ }^{60}$ Cf. lignes 9-10.

${ }^{61}$ Syntaxiquement cette forme est un gérondif, mais le signe final est $-u \check{s}$ alors qu'on attendrait $n=-4$ - - m. M. KHACHIKYAN (op. cit. n.33 pp. 261-62) avance différentes idées et remarques sur le suffixe $\xi$, intéressantes et également incohérentes. Elle note (p. 261) que $\breve{s}$ se rapproche dans son fonctionnement du suffixe $-b$ du prétérit archaïque et ressemble au suffixe $\check{s} s e$ de pronom relatif. Elle parle de «correlative particle». Plus loin (p. 262) elle s'égare en comparant $\breve{s}$ avec le suffixe de pluriel et en analysant zammalaštuš comme un "plural imperative [...] "may they be broken" [...] evidently connected with the element -š- of the pluralizing suffix $-a \check{s}^{\prime} »$. Mon 
"Que Tešsub brise la coupe, une fois arrachée l'applique brillante!

Analyse : u kudu karị pilleni elgilla sizeni!»

$U$ 'et puis', conjonction,

kudu $u^{62}$ 'briser', (?) à l'OPTA, ${ }^{63}$

kazi 'coupe', à l'ABS., sujet de kudu,

pilleni 'canal', à l'ABS. DEF., à valeur de LOC.,

elgilla 'applique brillante', à l'ABS. PL, sujet de kudu,

sizyeni 'eau', à l'ABS. DEF., à valeur de LOC., ${ }^{64}$

et que tombent la coupe dans le canal et l'applique dans l'eau!"

Traduction continue :

Tabrenni aluib billi ištani(yi)da:

Le fondeur se dit cette parole en son for intérieur:

"Iya(t) tabaliyaniš sidar(iy)a kaballiš ? "

"Pourquoi le cuivre que j'ai fondu me mandit-il??

U kari taballis sidarna kulurum:

Et puis le fondeur de mandire la coupe en répétant:

"Idiye kazi Te «̌subaš, elgi zammalaštuš

"Que Těšn brise la coupe, une fois arrachée l'applique brillante!

u kudu kazi pilleni elgilla šiyeni! »

et que tombent la coupe dans le canal et l'applique dans l'eau! »

\section{Les lignes 1-5 de la tablette $32.14 \mathrm{col}$. IV}

Le premier paragraphe du revers de la tablette (KBo 32.14 p.9) présente sensiblement cette apparence :

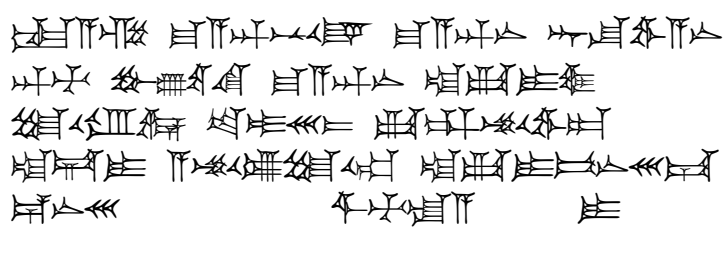

approche est que -um est un gérondif concomittant et $-u \check{s}$ un gérondif accompli.

${ }^{62}$ Logiquement la forme du verbe devrait être kudden. Neu (p. 158) oppose kudde(n) 'transitif' (ligne 11) et kudu 'intransitif' (ligne 57) mais (p. 159) parle d'Impératif, et non d'Optatif.

${ }^{63}$ Wegner (p. 232) analyse : « kud- „fallen” + u/o Modalformant. »

${ }^{64}$ Le mot hourrite siye est traduit par le mot hittite haba 'fleuve'. 


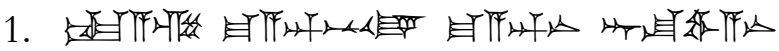
TC ka-a-zo ma-a-an-nu-u-bur ma-a-an-na tar-šu-wa-a-nə HCN kari mannubur manni taršuwani

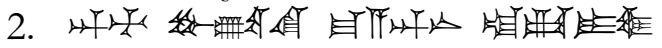

TC an-ti pu-ú-ut-ka ma-a-an-na at-ta-i-wo

$\mathrm{HCN}$ andi $\beta$ utki manni attaini

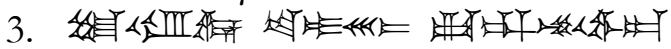

TC tu-u-ru-wa te-bé-eś-tab ta-al-mu-u-wa-ab ${ }^{65}$

HCN turuße tehestab talmuwab

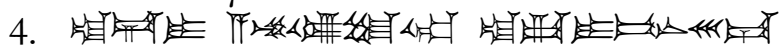

TC at-ta-i a-mu-u-lu-tu-u-um at at-ta-i-bi-na-eš-sa

$\mathrm{HCN}$ attai amuludum attaiwinešša

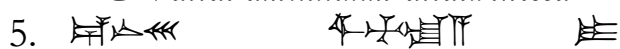

TC e-na-es šs si-ti-la- $a-\quad i$

$\mathrm{HCN}$ en $[n]$ es sidilai

Vocabulaire nouveau :

amul- 'regarder'

talmuw- 'devenir arrogant' < talmi 'grand, haut'

teh-est- 'grandir (énormément)'

turuße 'ennemi'

$\beta u t k i$ 'enfant, fils'

La segmentation en phrases est la suivante :

Kazi mannubur manni taršuwani.

Andi Butki manni attaini turuße.

Tehestab talmuwab attai amuludum, attaiwinešsa en $[n]$ ěs siddilai.

Analyse : Kazi mannubur manni taršuwani. ${ }^{67}$

Ce n'est pas une coupe, c'est un être humain.

Analyse: Andi Butki manni attaini turuße.

andi 'ce', à l'ABS., déterminant de $\beta u t k i$, Butki 'enfant, fils', à l'ABS., sujet de mann-, manni 'être',

attaini 'père', au GEN sans article, déterminant de turußi, turußi 'ennemi', à l'ABS., attribut,

Cet enfant est [devenu] ennemi de [son] père.

Analyse : Teheštab talmuwab attai amuludum,

teheštab 'grandir (énormément)', au PRET archaïque, talmuwab 'devenir arrogant', au PRET archaïque, attai 'père', à l'ABS. sans article, COD de amul-, amuludum 'regarder', au GER, avec NEG -ud-, ${ }^{68}$

\footnotetext{
${ }^{65}$ On peut noter l'absence de ligature entre les deux signes wa et $a$.

${ }^{66}$ On peut noter la présence de deux voyelles plene dans le même mot.

${ }^{67}$ Voir lignes 17 et 31.
} 
Il a beaucoup grandi, devint arrogant, ne considérant plus [son] père,

Analyse : attaiwinešša en [n]eš šidilai.

attaini-nešs- ${ }^{69}$ 'père', au GEN., déterminant de en $[n] e s ̌$, - a 'le', PEM, COD de šidil-, en [n]eš́ 'dieu', à l'ERG. sans article, sujet de šidil-, šidilai 'regarder', à l'INST., à valeur de GER, le dieu de son père le mandissant.

Traduction continue :

Kąi mannubur manni taršuwani. Ce n'est pas une coupe, c'est un être bumain.

Andi $\beta$ utki manni attaiwi turuße. Cet enfant est [devenu] ennemi de [son] père.

Tehestab talmuwab attai amuludum, Il a beaucoup grandi, devint arrogant, ne considérant plus [son] père, attaiwinešsa en [n]eš sidilai. le dieu de son père le maudissant.

\section{Les lignes 35-40 de la tablette 32.14 revers}

A partir de la ligne 23, le texte hourrite n'est plus écrit à la gauche du texte hittite sur deux colonnes séparées, mais sur toute la largeur, l'original hourrite étant suivi par la traduction en hittite. Cette mise en page différente explique que les numéros des lignes en hourrite sont discontinus dans les paragraphes qui suivent.

Le neuvième paragraphe du revers de la tablette (KBo 32.14 p.10) contient la onzième fable :

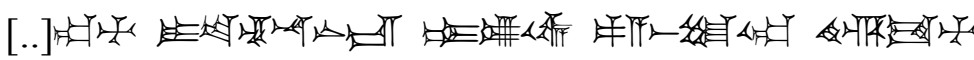

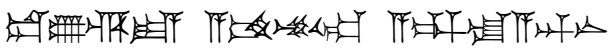

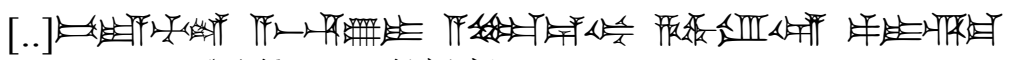

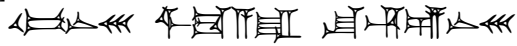

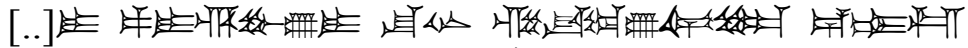

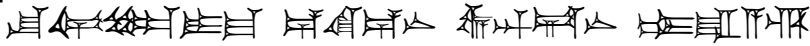

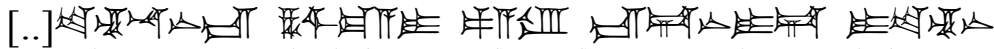

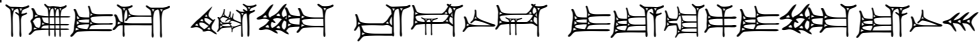

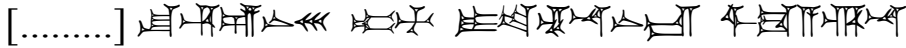

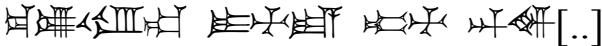

${ }^{68} \mathrm{Il}$ est fréquent que la négation $u d$ puisse se traduire mieux par 'ne plus' que par 'ne pas'. H. A. HOFFNER Jr (op. cit. n.3, p. 70) traduit d'ailleurs " and no longer looks at his father».

${ }^{69}$ attaini-nešs- a bien l'article défini mais en $[n] e s ̌ s$ est écrit avec un seul $n$. 


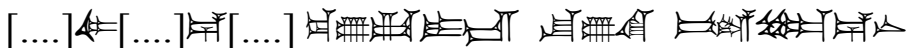 IF}

La tablette est légèrement abimée au début des lignes, mais la structure du texte et la comparaison avec d'autres paragraphes permet de reconstituer les signes manquants avec un haut niveau de plausibilité.

Un autre point est la présence de signes assez mal écrits et aussi de blancs au milieu de certains mots. $\mathrm{Neu}^{70}$ avance l'idée que le changement de mise en page : 2 colonnes avec chaque langue face à face $>1$ colonne avec le texte hittite après le texte hourrite, est destinée à simplifier la copie. Il est possible que la fin de la tablette ait été écrite par un scribe moins aguerri que le début. Le mot 'bâtisseur' est écrit tantôt idenni tantôt idennani. Neu (p.184) n'y voit pourtant pas une erreur (nichts Fehlerhaftes).

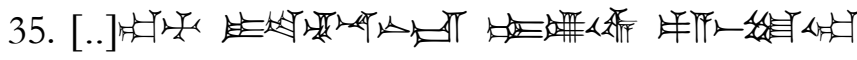
TC [ku]-um-ti i-te-en-na-na-is bé-lu-u-wo a pa-a-aś-tu-u-um HCN kumdi idennaniš heluwa pa[a]stum

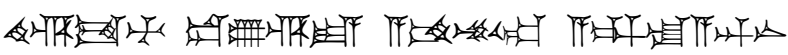
TC ha-ro-s $d^{71}-t i \quad d u-\hat{u}-r-i a$ a-am-mu-u-um a-al-la-an-nə $\mathrm{HCN}$ biritti turiya ammum Allanni

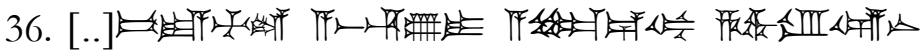
TC $[i]^{72}{ }^{72}$ bi-ia-ti-il a-aš-bu-ú-i a-la-e-u-ub ba-wo ${ }_{u}-$ ru-u-un-nə HCN išpiyatil ašbui aleub hawurunni

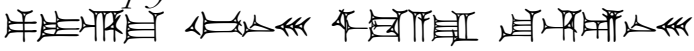

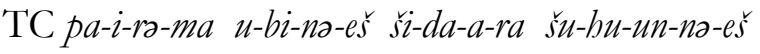
HCN pairima ußineš šidar(iy)a šubunnes

37. [..]

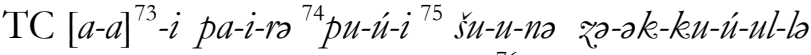
HCN ai pairi Buni šnni zakekulli ${ }^{76}$

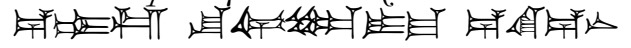
TC e-hé-ab- ${ }^{77}-s^{-} u-u l-l a-m a \quad e-k a-e-n a$ HCN ehepšsullima egen $(n) i$

70 (p. 179) «Ab Rs. 23 entfällt die bisherige Kolumnenanordnung ; der Schreiber [...], was für ihn sicherlich eine Arbeitserleichterung bedeutete. »

${ }^{71}$ Presque entièrement effacé : Neu propose de lire $i$ mais ad est meilleur car ce mot biritti est un emprunt akkadien.

72 Partiellement effacé : Neu propose de lire $i \check{s}$, ce qui semble probable.

73 Partiellement effacé : Neu propose de lire $a$ - $a$-, comme dans la ligne 48 de la colonne I.

${ }^{74} \mathrm{Il}$ manque un blanc ici entre les deux mots.

${ }^{75}$ Ce mot n'est pas écrit très lisiblement mais on peut le déduire de la ligne 47 de la colonne I.

${ }^{76} \mathrm{Neu}$ considère que ce verbe doit se lire qikekul-.

${ }^{77}$ Le mot est coupé en deux par un blanc. 


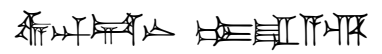

TC wa-an-ta-na bé-ra-a-ro

HCN Bandani herari

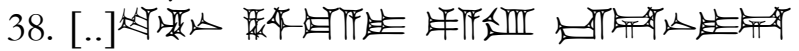

TC [i]-te-en-na ba-si-ma-a-i pa-a-ru iš-ta-na-i-ta

HCN idenni hašimai paru b7 ištanida

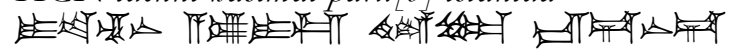

TC i-te-en-na a-lu-i-ab ba-il-ba ${ }^{78}$ is-ta-na-ta

HCN idenni aluib billi istanida

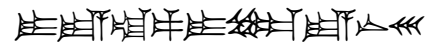

TC i-ia-at ${ }^{79}$ pa-i-lo-ia-na-is

$\mathrm{HCN}$ iyat [ta] pailiyanis

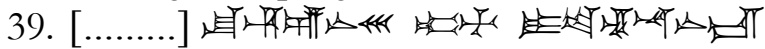

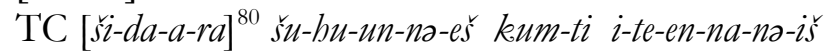

HCN sidariya šubunneš kumdi idennaniš

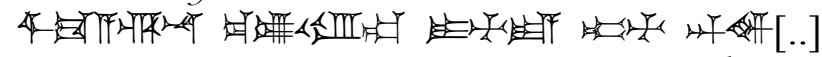

TC ši-da-a-ar-na ku-lu-u-ru-um i-ti-ia kum-ti ${ }^{\mathrm{d}} \mathrm{IM}$-[as']

HCN sidarna kulurum idiya kumdi ${ }^{\mathrm{d}}$ Teřšnbaš

40. $[\ldots . .].[\ldots].[\ldots$.

TC $\left.[\text { ra-am- }]^{81}-m i-[i-i a \quad t e]-e-[l]\right]^{82} \quad k u-u-t a-i-e s^{2}$

HCN [ram]mi[ya te]e[li] kudais

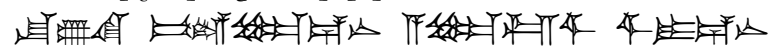

TC $\check{s u-u ́-k a}$ bi-il-la-e-na a-la-ab-sis $\quad \grave{s i}-i-e-n a$

HCN šugi pillini alipši šiyeni

Vocabulaire nouveau :

ale- 'faire monter'

Allani 'Allani', déesse du monde souterrain,

ašbui 'vers le haut'

edenni 'bâtisseur, architecte' < akkadien iținnu, ${ }^{83}$

bawurni 'ciel'

biritti 'fondation' < akkadien biritu 'creusement', ${ }^{84}$ išpiyati 'créneau' ${ }^{85}$

${ }^{78}$ Sans le signe $a b$ de la ligne 52 col. I.

${ }^{79} \mathrm{Il}$ manque un blanc ici entre les deux mots.

${ }^{80}$ Effacé mais très probable.

${ }^{81}$ Effacé. Neu ne propose rien mais d'après la ligne 57 colonne 1 qui contient zammalaštus, on peut supposer que celle-ci contient zamm-iya.

82 Effacé. Neu ne propose rien mais le texte hittite semble indiquer un mouvement vers le haut: teli est donc envisageable. La ligne 57 colonne 1 contient elgi, mais cela ne semble pas convenir.

${ }^{83} \mathrm{Neu}$ (p. 183) écrit itinnu, etennu mais le hourrite idenni avec $d$ ne peut pas dériver d'une sourde. A noter que l'emphatique de l'akkadien est rendue par une sonore en hourrite, un phénomène récurrent.

${ }^{84} \mathrm{Neu}$ (p. 185) a bien vu que ce mot est akkadien mais il lit * biridi en hourrite, ce qui est incompatible avec le principe des simples valant sonores en cunéiforme anatolien.

${ }^{85}$ Ce mot fait penser à išpanti 'carquois'. 
kumdi 'tour'

paašt- 'construire' 86

pairi 'constructeur'

šugi 'toit' < (?) akkadien šqu 'hauteur',

šubni 'mur'

La segmentation en phrases est la suivante :

Kumdi idennaniš beluwa pa $[a]$ štum,

biritti turiya ammum Allanni,

išpiyatil ašbui aleub hawurunni.

Pairima ußines šidar(iy) a šubunnes :

" ai pairi ßuwi šuni zok.kulli, ehepšullima egen(n)i ßandani herari. »

Idenni hašimai paru [b] ištanida.

Idenni aluib hilli ištanida :

"Iyat[ta] pailiyaniš sidariya šubunnes ? "

Kumdi idennanis sidarna kulurum :

"Idiya kumdi ${ }^{d}$ Těšsubaš !

[ram]mi[ya te]e[li]!

kudaiš sugi pillini, alipši šiyeni! 》

Analyse : Kumdi idennanǐ̌ beluwa pa[a]štum, ${ }^{87}$

kumdi 'coupe', à l'ABS., COD de paašt-,

idennaniš 'bâtisseur', à l'ERG. DEF., sujet de paašt-,

beluwa 'gloire', au DAT.,

pa $[a]$ štum 'fondre', au GER,

Une tour le bâtisseur ayant construite pour (sa) réputation,

Analyse : biritti turiya ammum Allanni,

biritti 'fondation', à l'ABS., COD de amm-,

turiya 'vers le bas',

ammum 'mener, aller', au GER,

Allanni 'Allani', à l'ABS. DEF., à valeur de LOC.,

les fondations allant vers le bas jusqu'à Allani,

Analyse : išpiyatil ašbui aleub hawurunni.

išpiyatil[la] 'créneau', à l'ABS. PL., COD de ale-, ašbui 'vers le haut', aleub 'élever', au PRET archaïque, hawurunni 'ciel', à l'ABS. DEF., à valeur de LOC., il avait élevé les créneaux haut dans le ciel.

Analyse : Pairima ußineš šidar(iy)a šubunneš :

pairi- 'constructeur', à l'ABS. sans article, COD de šidar-, -ma 'et', conjonction ENC,

ußineš 'insensé', à l'ERG. DEF., déterminant de šubni,

sidar(iy)a 'maudire',

šubunneš 'mur', à l'ERG. DEF., sujet de šidar-,

Et le mur insensé de mandire le constructeur:

\footnotetext{
${ }^{86} \mathrm{Il}$ est possible que ce mot soit bisyllabique *[pa'ast-].

87 Parallèle à la ligne 42 colonne 1 : Kazi taballiš heluwa taßaštum.
} 
Analyse : "ai pairi Buwi šuni zokkulli,

"[Et,] constructeur, si ta main se brise,

Analyse : ehepšullima egeni ßandani herari!»

[alors] que [ton] tendon interne droit se coince! ${ }^{88}$

Analyse : Idenni hasimae paru istani(yi)da. ${ }^{89}$

Le bâtisseur, en entendant [cela], se mit en colère en son [for] intérieur.

Analyse : Idenni aluib billi istanida: ${ }^{90}$

Le bâtisseur se dit cette parole en son for intérieur:

Analyse : "Iyat pailiyaniš šidar(iy)a šubunneš ? 》 ${ }^{91}$

"Pourquoi le mur que j'ai construit me mandit-il? 》

Analyse : Kumdi idennanišs sidarna kulurum : ${ }^{92}$

Le bâtisseur de mandire la tour en répétant:

Analyse : "Idiya kumdi ${ }^{d}$ Tešsubaš ! $^{93}$

"Que Tešsub brise la tour!

Analyse : [zam $]$ mi[ya te]e[l] $!^{94}$

"Qu'il l'envoie dans les airs!

Analyse : kudaiš šugi pillini, alipšsi šiyeni! » ${ }^{95}$

kudais' 'tomber', au GER., ${ }^{96}$

šugi 'toit', à l'ABS. sans article, COD de kud-,

pillini 'canal', à l'ABS. DEF., à valeur de LOC.,

alipši 'brique', à l'ABS. sans article, COD de kud-, ${ }^{97}$

sizyeni 'mur', à l'ABS. DEF., à valeur de LOC.,

après avoir fait tomber le toit dans le canal et les briques dans le flewve!»

Traduction continue :

Kumdi idennaniš heluwa pa[a]šstum,

Une tour le bâtisseur ayant construite pour (sa) réputation,

biritti turiya ammum Allanni,

les fondations allant vers le bas jusqu'à Allani,

išpiyatil ašbui aleub hawurunni.

il avait élevé les créneaux haut dans le ciel.

${ }^{88}$ Voir les lignes 17-49 colonne 1.

${ }^{89}$ Voir les lignes 8 et 50-51 colonne 1.

${ }^{90}$ Voir la ligne 52 colonne 1.

${ }^{91}$ Voir les lignes 53-54 colonne 1.

${ }^{92}$ Voir les lignes 54-55 colonne 1.

${ }^{93}$ Voir la ligne 56 colonne 1.

${ }^{94}$ Voir la ligne 57 colonne 1.

${ }^{95}$ Voir les lignes 57-59 colonne 1.

${ }^{96}$ Cf. rammalast-us.

${ }^{97}$ Le mot est singulier mais le sens est pluriel. Neu (p. 195) interprète la finale $-s \grave{i}$ comme un suffixe de formation d'abstraits. 
Pairima ußineš šidar(iy) a šubunneš :

Et le mur insensé de mandire le constructeur :

"ai pairi Buwi šnni zakkeulli,

«Et,] constructeur, si ta main se brise,

ehepšullima egeni ßandani herari!»

[alors] que [ton] tendon interne droit se coince!»

Idenni hasimae paru ištani(yi)da.

LLe bâtisseur, en entendant [cela], se mit en colère en son [for] intérieur.

Idenni aluib billi istanida:

Le bâtisseur se dit cette parole en son for intérieur:

"Iyat pailiyaniš šidar(iy)a šubunneš ? 》

"Pourquoi le mur que j'ai construit me mandit-il?"

Kumdi idennaniš sidarna kulurum :

Le bâtisseur de maudire la tour en répétant:

"Idiya kumdi 'Teššbaš!

"Que Tešsub brise la tour!

[ram]mi[ya te]e[li]!

"Qu'il l'envoie dans les airs!

kudaiš sugi pillini, alipši sizyeni!»

après avoir fait tomber le toit dans le canal et les briques dans le fleuve!»

Certaines formes sont un peu étranges. Si on admet que les formes idenni (Abs.) idennanis (Erg.) sont correctes, on peut noter que le suffixe d'Erg. -niš ne se suffixe pas à la base idenn(i), comme cela serait attendu, mais à une base allomorphe idenna qui ressemble à l'accusatif akkadien, ce qui fait que l'alternance en hourrite entre idenni (Abs.) idennaniš (Erg.) est en quelque sorte parallèle à celle de l'akkadien idennu (Nom.) idenna (Acc.). Cela incite à conclure que cette parabole a été écrite ou traduite dans un environnement qui devait être bilingue, ou en tout cas pour qui la morphologie akkadienne était très familière et pouvait faire intrusion dans celle du hourrite.

Plusieurs verbes ont des sortes de diphtongues pré-suffixales qu'on n'observe pas d'habitude : al-eu-b, al-ui-b, kud-ai-s. Le suffixe d'Optatif $i d-i y a$ est peut-être plus archaïque que la forme $i d$-iye, vue à la ligne 56 colonne 1 .

Au final cette parabole semble présenter des formes dialectales et archaïsantes et une influence importante de l'akkadien sur le lexique et même la morphologie.

\section{Les lignes $48-49$ de la tablette 32.14 revers}

Le onzième paragraphe du revers de la tablette (KBo 32.14 p.10) comprend le texte suivant : 


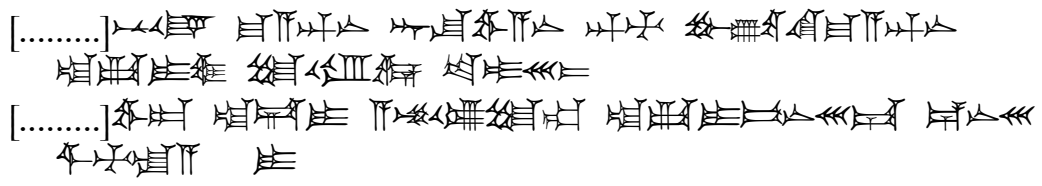

Ce paragraphe réassemble des éléments déjà vus plus haut, qui expliquent comment il convient d'interprêter les lignes 35-40:

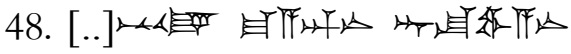

TC [ku-um-ti ma-a-an-]nu-u-bur ma-a-an-no tar-šu-wa-a-nə

HCN kumdi mannubur manni taršuwani

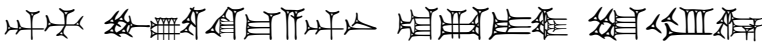

TC an-ti pu-u-ut-ko-ma-a-an-no at-ta-i-wo, tu-u-ru-wo,

$\mathrm{HCN}$ andi $\beta$ utki-manni attaini turu $\beta$ e

W

TC te-hé-eŕs-tab

HCN tehestab

49. [..]

TC [ta-al-mu-u-]wo-ab at-ta-i a-mu-u-lu-tu-um

HCN talmuwab attai amuludum

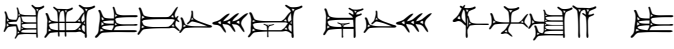

TC at-ta-i-bi-no-ers-ša e-no-ers šs-ti-la-a- i

$\mathrm{HCN}$ attaiwine ̌̌ša en $[n]$ es sidilai

Vocabulaire nouveau : aucun. Analyse : voir $\$ 8$.

Traduction continue :

Kumdi mannubur manni taršuwani.

Ce n'est pas une tour, c'est un être bumain.

Andi ßutki manni attaiwi turuße.

Cet enfant est [devenu] ennemi de [son] père.

Tehestab talmuwab attai amuludum,

Il a beaucoup grandi, devint arrogant, ne considérant plus [son] père,

attaiwinešsa en $[n]$ ě́ šidilai.

le dieu de son père le maudissant.

\section{Les lignes 55-61 de la tablette 32.14 revers}

Le quatorzième paragraphe du revers de la tablette (KBo 32.14 p.11) est le dernier qui soit en bon état de conservation. Il est relativement lisible en intégralité. A contrario la traduction en hittite, qui de surcroit est écrite sur la tranche de la tablette, est très endommagée.

Il comprend le texte suivant : 


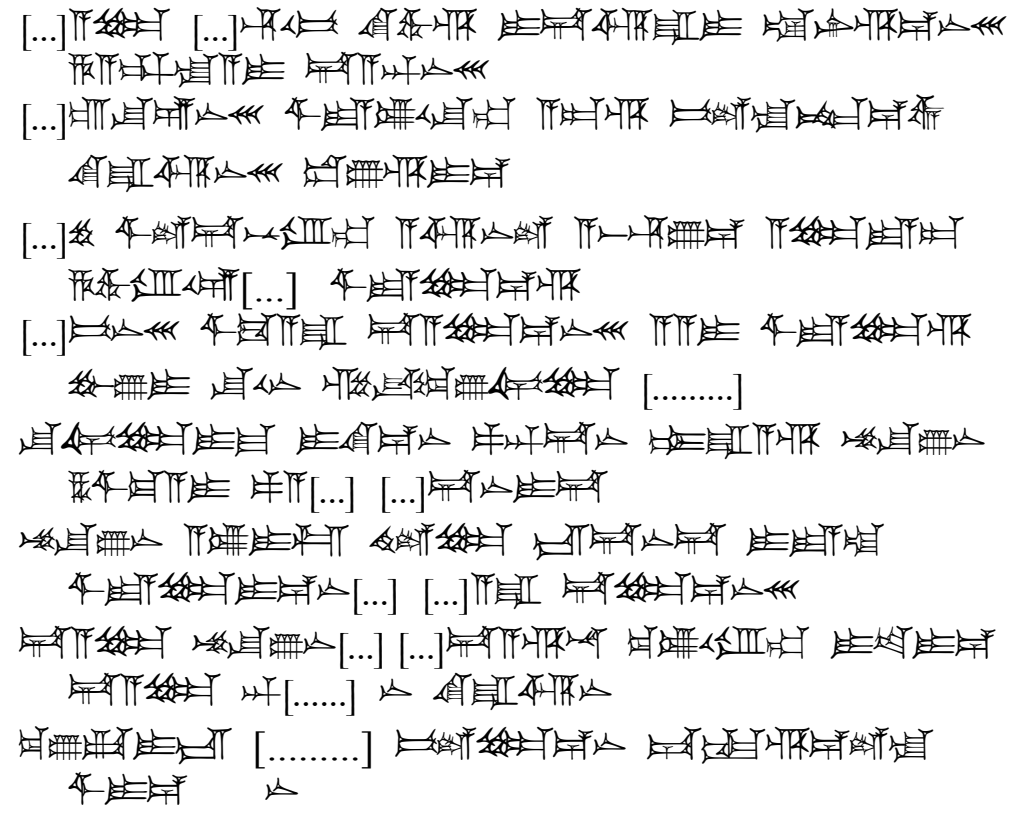

La structure du paragraphe est sensiblement la même que celle du \$18. vu ci-dessus. Le thème et le vocabulaire sont légèrement différents. Neu a été handicapé dans son livre par l'absence de traduction et de contrepartie hittite au texte hourrite. L'analyse qui suit est donc en partie inédite.

55. [...] [1 [...

TC [ta]-a-la ša $a^{98}-b u-u-b i \quad \check{s} a-s ̌ a r-r i \quad k a-w \partial_{u}-r a$

HCN tali šahu-ubi šašarri kimuri[b]

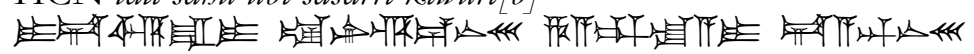

TC i-ta-ar-ra-i za-ar-re-e-na-eš ha-a-al-la-a-i ša-a-an-nə-e ̌́

HCN idarrai zerreneš hallai sanneš

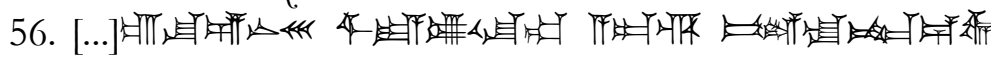

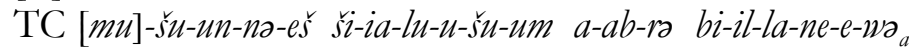

HCN musunneš sizyalušm abri pillannewa

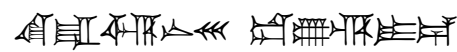

TC ka-ra-ar-na-eš du-ú-ra-i-e

HCN kirarnes turiye

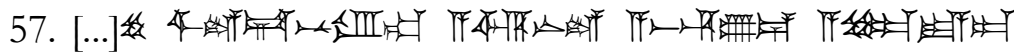

TC [e]-še ši-il-ta-nu-ru-um a-ar-no-il a-aš-bu-ú-e a-la-ya-ab

HCN eše sildanurum arnil[la] ašbue aleyab

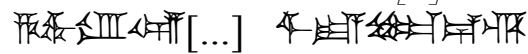

TC ha-wor-ru-u-un-[nə] siz-ia-la-e-ro

HCN hawurunni sizyaleri

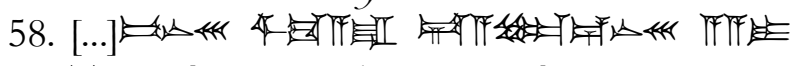

TC [u]-bi-na-eš si-da-a-ra ta-a-la-e-nə-ers a-a-i

HCN ußineš šidar(iy) a taleneš ai

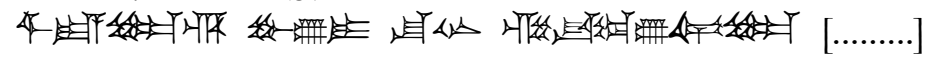

${ }^{98}$ Le signe est abimé : 5 a ou bien opte pour tahubi sans proposer de sens, tout en mentionnant l'ambiguïté. 
TC ši-ia-la-ra ${ }^{99} p u-\dot{u}-i \quad \dot{s} u-u-n a$ za-ak-ku-úul-la [e-hé-ab]-

$\mathrm{HCN}$ sizaleri ßuwi šuni zakekulli ${ }^{100}$ ehep-

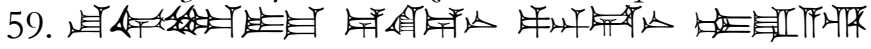

TC -šu-ul-la-ma ${ }^{101}$ e-ka-e-na pa-an-ta-na bé-ra-a-ro

HCN -sullima egen (n)i $\beta$ andani herari

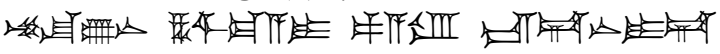

TC mu-šsu-un-nə ha-ši-ma-a-i pa-a-ru iš-ta-nə-i-ta

HCN mušunni hašimai paru[b] istanida

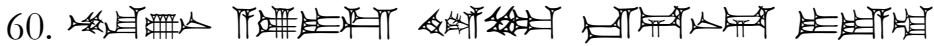

TC mu-šs-un-na a-lu-i-ab ba-il-la ${ }^{102}$ iś-ta-na-ta i-ia-at

HCN musunni aluib billi istanida iyat [ta]

4-

TC ši-ia-la-i-e-no-[as] [risi-da-] $a-r a^{103}$ ta-la-e-no-eš

HCN sizaliyenes sidariya talenes

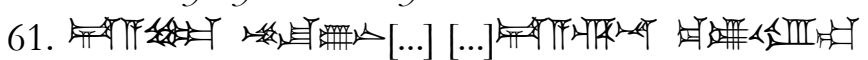

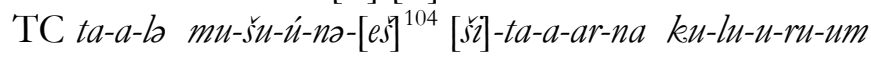

HCN tali mušrunneš sidarna kulurum

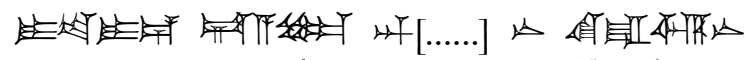

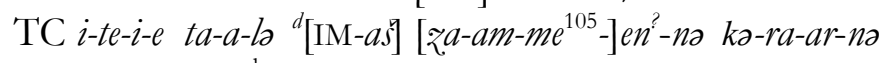

HCN ideye tali ${ }^{\mathrm{d}}$ Tešsubaš zammenni kirarni

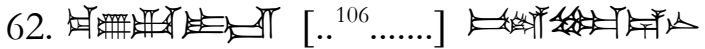

TC ku-ú-ta-i-ǐs $\left[a-a r^{?}-n a^{?}-i l^{2}\right]^{107}$ bi-il-la-e-na

HCN kudais arnil[la] pilleni

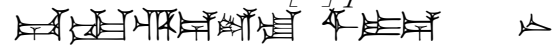

TC $\check{s} a-k a-r a-e-i l-l a r i s i-i-e \quad-n a$

HCN šagarella šizeni

Vocabulaire nouveau :

abri 'tas, pile de bois'

arni 'pointe, bout de branche'

hall- 'aller chercher', cf. hourrite ba- 'prendre',

kißur- 'poser', cf. hourrite kiß- 'déposer, mettre',

kirari 'fondation' < akkadien karäru 'jeter (les fondations)', mušuni 'empileur (de bois)', cf. hourrite muš- 'ajuster, faire bien',

${ }^{99} \mathrm{Il}$ manque un blanc ici entre les deux mots.

${ }^{100}$ Les trois premiers signes de ce mot sont abimés.

${ }^{101}$ Le mot est coupé en deux sur deux lignes.

102 Sans le signe $a b$ de la ligne 52 col. I.

${ }^{103}$ Effacé mais très probable.

104 Partiellement effacé.

${ }^{105}$ L'espace entre les signes $<^{d}>$ et $<e n^{2}>$ est court (3/4 signes), ce qui signifie que $\mathrm{d}^{T}$ Těšsubaš est écrit $<\mathrm{d} I \mathrm{M}>$ ou, dans le cas contraire, que le verbe n'est pas zamm-.

106 Au début de la zone effacée on distingue un signe $\mathbb{T} a$.

107 Après kirarni (ligne 56) vient arnil (ligne 57), ce qui est cohérent avec la présence d'un signe $\prod_{a} a$ dans cette ligne. 
šagare 'lichen' [ou peut-être 'bourgeon, brindille']

šabu 'cochon' < akkadien šabu,

šani 'coursier' < akkadien šànu,

šašari 'scie' < akkadien ša ššăru 'scie',

sizal- 'installer'

sildanur- 'toucher'

zerre 'âne', ${ }^{108} \mathrm{cf}$. akkadien sirrimu 'onagre, âne sauvage',

La segmentation en phrases est la suivante :

Tali šabu-ubi šašarri kißuri[b], idarrai zerreneš hallai šanneš.

Mušunneš siyalušum abri pillannewa,

kirarneš turiye è̃e šildanurum, arnil[la] ašbue aliyab hawurunni.

Siyaleri ußineš sidar(iy) a taleneš :

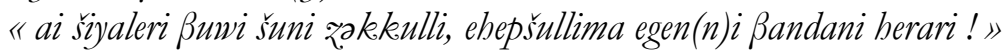

Mušnni bašimai paru[b] ištanida.

Mušunni aluib billi ištanida :

"iyat[ta] sizyaliyeneš sidar(iy)a taleneš ? 》

Tali mušunneš šidarna kulurum:

"Ideye tali ${ }^{\mathrm{d}}$ Teššbušs! Zammenni kirarni!

Kudaiš arnil[La] pilleni, šagarella šiyeni!»

Analyse : Tali šabu-ubi šašarri kißuri[b],

tali 'bois', à l'ABS., COD de kißur-,

šahu-ubi 'cochon stupide', à l'ABS. sans article, sujet de kißur-, ${ }^{109}$

šašarri 'scie', à l'ABS. DEF., à valeur de LOC., ${ }^{110}$

kißßuri[b] 'poser', au PRET archaïque,

Du bois un cochon stupide posait sur la scie,

Analyse : idarrai zerreněs hallai sanneš.

idarrai 'casser', à l'INST., à valeur de GER, zerrenes 'âne', à l'ERG. DEF., sujet de idarr-, hallai 'aller chercher', à l'INST., à valeur de GER, šanneš' coursier', à l'ERG. DEF., sujet de hall-, un âne le cassant en petit bois, un coursier allant le chercher.

Analyse : Mušunneš šzyalušum abri pillannewa,

mušunneš ‘empileur', à l'ERG. DEF., sujet de šiyalušs-, sizyalušm 'ranger', au GER avec suffixe intensif - $u s^{\prime}$, abri 'tas, pile de bois', à l'ABS., COD de šiyaluš, pillannewa 'canal', au DAT. DEF., à valeur de LOC., L'empileur rangeant une pile de bois près du canal,

108 Traduit AN-ŠE en hittite.

${ }^{109}$ Le mot n'est pas à l'ergatif, bien que le verbe soit transitif, et c'est le seul être animé, donc sémantiquement le seul sujet possible. Il est possible que la finale d'ergatif $-i \check{s}$ manque à cause de l'initiale du mot suivant.

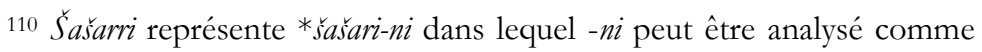
l'article au cas absolutif défini ou comme une marque de cas locatif archaïque. 
Analyse : kirarnes turiye eše šildanurum, ${ }^{111}$

kirarneš ‘fondation', à l'ERG. DEF., sujet de šildanur-, turiye 'vers le bas', eše 'terre', à l'ABS. sans article, à valeur de LOC., silldanurum 'canal', au DAT. DEF., à valeur de LOC., la fondation touchant par terre,

Analyse : arnil[la] ašbue aliyab hawurunni. ${ }^{112}$ arnil[la] 'pointe', à l'ABS. PL., sujet de aliy-, ašbue 'vers le haut', aleyab 'élever', au PRET archaïque intransitif, hamurunni 'ciel', à l'ABS. DEF., à valeur de LOC., les pointes s'élevaient haut dans le ciel.

Analyse : Šiyaleri ußineš šidar(iy) a taleneš : ${ }^{113}$ Et le bois insensé de mandire le rangeur :

Analyse : «ai sizyaleri $\beta$ uwi šnni zakkeulli, ${ }^{114}$ «[Et, le] rangeur, si sa main se brise,

Analyse : ehepšullima egeni Bandani herari! » ${ }^{115}$ [alors] que son tendon interne droit se coince! »

Analyse : Mušunni hašimae paru ištani(yi)da. ${ }^{116}$ L'empileur, en entendant [cela], se mit en colère en son [for] intérieur.

Analyse : Mušunni aluib billi ištanida: ${ }^{117}$ L'empileur se dit cette parole en son for intérieur:

Analyse : «Iyat [ta] sizaliyeneš sidar(iy)a taleneš ? 》 ${ }^{118}$ "Pourquoi le bois que j'ai rangé me maudit-il ?"

Analyse : Tali mušunneš šidarna kulurum : ${ }^{119}$ L'empileur de maudire le bois en répétant:

Analyse : «Ideye tali ${ }^{\mathrm{d}}$ Tě̌šbaš ! ${ }^{120}$ "Que Tešsub brise le bois!

Analyse : Zammenni kirarni! ${ }^{121}$ "Qu'ils détruisent la fondation!

\footnotetext{
111 Cf. Ligne 35 revers.

${ }^{112}$ Cf. début de la ligne 36 revers.

113 Cf. fin de la ligne 36 revers.

114 Cf. Ligne 37 revers.

115 Voir les lignes 17-49 colonne 1 et 37 revers.

116 Voir les lignes 8, 50-51 colonne 1 et 38 revers.

117 Voir la ligne 52 colonne 1 et 38 revers.

118 Voir les lignes 53-54 colonne 1, et 38-39 revers.

119 Voir les lignes 54-55 colonne 1 et 39 revers.

${ }^{120}$ Voir la ligne 56 colonne 1 et fin de la ligne 39 revers.

${ }^{121}$ Voir la ligne 57 colonne 1 et 40 revers.
} 
Analyse : kudaiš arnil[la] pilleni, šagarella šiyeni!» ${ }^{122}$

kudais 'tomber', au GER., arnil[la] 'pointe', à l'ABS. PL., COD de kud-, pilleni 'canal', à l'ABS. DEF., à valeur de LOC., arnil[la] 'pointe', à l'ABS. PL., COD de kud-, sagarella 'lichen', ${ }^{23}$ à l'ABS. PL., COD de kud-, sizyeni 'mur', à l'ABS. DEF., à valeur de LOC.,

après avoir fait tomber le bout des branches dans le canal et les lichens dans le fleuve!"

Traduction continue :

Tali šabu-ubi šašarri kißuri[b],

Du bois un cochon stupide posait sur la scie,

idarrai zerreneš hallai šanneš.

un âne le cassant en petit bois, un coursier allant le chercher.

Mušunneš sizyalusum abri pillannewa,

L'empileur rangeant une pile de bois près du canal,

kirarneš turiye eše sildanurum, la fondation touchant par terre,

arnil[La] ašbue aliyab hawurunni.

les pointes s'élevaient haut dans le ciel.

Šiyaleri ußineš šidar(iy) a taleneš :

Et le bois insensé de mandire le rangeur :

" ai šiyaleri ßuwi šnni zokkulli,

$"[E t$, le] rangeur, si sa main se brise,

ehepšullima egeni ßandani herari! »

[alors] que son tendon interne droit se coince! »

Mušunni hašimae paru ištani(yi)da.

L'empileur, en entendant [cela], se mit en colère en son [for] intérieur.

Mušunni aluib billi ištanida:

L'empileur se dit cette parole en son for intérieur :

"Iyat[ta] siyaliyeneš šidar(iy)a taleneš ? "

"Pourquoi le bois que j'ai rangé me mandit-il ? "

Tali mušunneš šidarna kulurum:

L'empileur de maudire le bois en répétant:

"Idiya tali ${ }^{\mathrm{d}}$ Těšsubaš!

"Que Tešsub brise le bois!

Zammenni kirarni!

"Qu'ils détruisent la fondation!

122 Voir les lignes 57-59 colonne 1 et 40 revers.

${ }^{123} \mathrm{Neu}$ (p.212) explique préférer traduire šagare par Moosflechten 'lichen', plutôt que par Knospe, Trieb, Schößling 'bourgeon, rejeton', qui est la traduction qu'avait retenue Catsanicos (op. cit. n. 5). 
kudaiš arnil[la] pilleni, šagarella šiyeni!»

après avoir fait tomber le bout des branches dans le canal et les lichens dans le fleuve!»

Il est intéressant de noter que dans la première phrase un mot sur deux est un emprunt akkadien. La suite est néanmoins dans la continuité du $₫$ 9.

\section{Conclusion}

L'article s'est intéressé à plusieurs fables contenues dans la tablette KBo 32.14, qui fait partie d'un lot de documents mis au jour lors des campagnes de fouilles en 1983 et 1985 à Boğazköy. Les tablettes, publiées dans le fascicule $\mathrm{KBo} 32$, sont bilingues et écrites à la fois en hourrite et en hittite. En général, on s'accorde à mettre spécialement en avant leur très grande valeur pour la connaissance et la compréhension du hourrite, qui étaient restées handicapées par la difficulté de textes uniquement monolingues.

Mais l'intérêt de ces tablettes n'est pas seulement grammatical ou lexical. Car elles offrent une fenêtre sur un genre littéraire, très peu attesté dans les découvertes en langue akkadienne, à savoir les fables d'Esope. On sait que ce type de fables était très populaire en Mésopotamie, mais il fut volontairement ignoré par les scribes.

Un autre intérêt est de donner une profondeur littéraire et historique à certains passages de la Bible. Ainsi, on peut comparer un extrait des lignes $42-51$ de la tablette 32.14 col. I avec Esaïe :

Une coupe le fondeur ayant coulée pour (sa) réputation, et la coulant en la formant bien [et lui] appliquant une couche brillante, et puis il cisela des ciselures et [lui] donna de l'éclat.

Et le cuivre insensé de mandire le fondeur : "Fondeur, si ta main se brise, [alors] que [ton] tendon interne droit se coince!»

Le fondeur, en entendant [cela], se mit en colère en son [for] intérieur.

Esaïe 49:9 : "Malheur à qui, cruchon parmi les cruchons de glaise, chicanerait celui qui l'a formé ! L'argile dira-t-elle à celui qui lui donne forme : «Que fais-tu ?», et l'oeuvre réalisée par toi dirat-elle : «Il n'a pas de main »?

Il est très probable que les images utilisées par Esaïe devaient avoir une très grande résonance parmi la population, qui était très familière avec elles par ailleurs, depuis plusieurs siècles au moins.

Abstract: The paper analyzes the Fables in the tablet KBo 32.14. It is first argued that the tablets containing Fables should be dealt with as a different work from the Song of Liberation of Ebla. All the tablets, found in 1983 and 1985 in Boğazköy, are bilingual and therefore extremely valuable for the understanding 
of Hurrian. But they also offer a unique window on a literary genre, very little attested in Akkadian language tablets, i.e Aesopic Fables. This genre was very popular in Mesopotamia, but ignored by scribes. In addition, the theme of the object revolting against its creator, reflected in the Bible as clay rebelling against the potter, is interestingly developed in some Fables, that were probably widely known in the ancient Near-East. 